\title{
ARTICLE
}

\section{The recruitment of extra-intestinal cells to the injured mucosa promotes healing in radiation enteritis and chemical colitis in a mouse parabiosis model}

\author{
J. Sung ${ }^{1}$, C. P. Sodhi' ${ }^{2}$, L. Voltaggio ${ }^{3}$, X. Hou ${ }^{4}$, H. Jia ${ }^{2}$, Q. Zhou ${ }^{2}$, D. Čiháková ${ }^{3}$ and D. J. Hackam ${ }^{1,2}$
}

\begin{abstract}
Mucosal healing occurs through migration and proliferation of cells within injured epithelium, yet these processes may be inadequate for mucosal healing after significant injury where the mucosa is denuded. We hypothesize that extra-intestinal cells can contribute to mucosal healing after injury to the small and large intestine. We generated parabiotic pairs between wild-type and tdTomato mice, which were then subjected to radiation-induced enteritis and 2,4,6-trinitrobenzene sulfonic acid (TNBS)-induced colitis. We now show that as compared with singleton mice, mice with a parabiotic partner were protected against intestinal damage as revealed by significantly reduced weight loss, reduced expression of pro-inflammatory cytokines, reduced enterocyte apoptosis, and improved crypt proliferation. Donor cells expressed CD45 ${ }^{-}, \mathrm{Sca}-1^{+}, \mathrm{c}_{\mathrm{kit}}{ }^{+}$, and CXCR4 ${ }^{+}$and accumulated around the injured crypts but did not transdifferentiate into epithelia, suggesting that extra-intestinal cells play a paracrine role in the healing response, while parabiotic pairings with Rag $1^{-/-}$mice showed improved healing, indicating that adaptive immune cells were dispensable for mucosal healing. Strikingly, ablation of the bone marrow of the donor parabionts removed the protective effects. These findings reveal that the recruitment of extra-intestinal, bone marrow-derived cells into the injured intestinal mucosa can promote mucosal healing, suggesting novel therapeutic approaches for severe intestinal disease.
\end{abstract}

Mucosal Immunology (2019) 12:503-517; https://doi.org/10.1038/s41385-018-0123-3

\section{INTRODUCTION}

Healing from mucosal injury is of fundamental importance to gastrointestinal homeostasis. ${ }^{1}$ Current dogma suggests that mucosal healing occurs through two parallel processes, which include restitution, i.e. the migration of healthy cells adjacent to the site of epithelial disruption towards to epithelial defect, ${ }^{2}$ and proliferation, i.e. the generation of new epithelial cells from progenitor cells located within the crypts of Lieberkuhn. ${ }^{3}$ Importantly, clinical observations suggest that these two processes may be inadequate to account for the mucosal healing that occurs in the setting of severe mucosal injury, implying that additional, unexplored healing processes may play a role. For instance, in the setting of radiation injury to the intestine, a loss of intestinal stem cells occurs, yet the intestinal mucosa still recovers. ${ }^{4-6}$ Further, in the setting of advanced inflammatory bowel disease, marked denudation of the mucosal epithelium occurs, yet epithelial healing is still achieved, even though the adjacent epithelial cells may be completely lost. ${ }^{7}$ Such observations raise the possibility that previously unrecognized pathways could play key roles in intestinal mucosal healing after mucosal injury, especially under conditions in which proliferation and restitution are unlikely to be effective. ${ }^{8-11}$ In this regard, previous authors have suggested the possibility that circulating, extraintestinal cells can migrate to sites of intestinal injury where they may contribute- either directly or in a paracrine fashion-to the mucosal healing response. ${ }^{12,13}$ However, the lack of reliable models, and inconsistency in the severity of intestinal injury, have yielded discrepant findings, ${ }^{14-16}$ so that the role of extra-intestinal cells to intestinal mucosal healing remains an open question. ${ }^{17,18}$

To address this gap in our knowledge, we have now employed a parabiosis system to establish a shared circulation between mice who were then subjected to either radiation-induced enteritis or chemically induced colitis in order to investigate whether extraintestinal cells can participate in the repair of injured intestine. ${ }^{19,20}$ We now report that circulating cells migrate to the sites of injury, are incorporated into the injured mucosa, and play a critical and previously unrecognized role in healing after mucosal injury in two complementary models.

\section{RESULTS}

Development of a shared circulatory system in parabiotic mouse pairs

To directly evaluate the involvement of extra-intestinal cells in intestinal mucosal repair, we established a parabiotic mouse model. To do so, we surgically connected wild-type mice to either wild-type or transgenic ROSA26-CAG-tdTomato mice, which were developed as shown in Fig. 1a to constitutively express the red fluorescence reporter protein TdTomato in all cells, thus allowing us to track the exchange of cells from uninjured to injured mice.

\footnotetext{
'Institute of Genetic Medicine, School of Medicine, Johns Hopkins University, Baltimore, MD, USA; ${ }^{2}$ Division of Pediatric Surgery, Johns Hopkins Children's Center and Department of Surgery, Baltimore, MD, USA; ${ }^{3}$ Department of Pathology, Johns Hopkins University School of Medicine, Baltimore, MD, USA and ${ }^{4}$ W. Harry Feinstone Department of Molecular Microbiology and Immunology, Bloomberg School of Public Health, Johns Hopkins University, Baltimore, MD, USA

Correspondence: D. J. Hackam (dhackam1@jhmi.edu)
}

Received: 1 July 2018 Revised: 5 November 2018 Accepted: 20 November 2018

Published online: 7 January 2019 


\section{a}
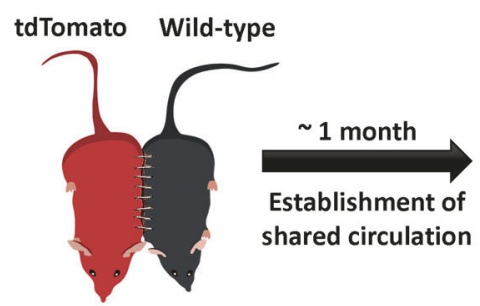
Establishment of
shared circulation

\section{b}

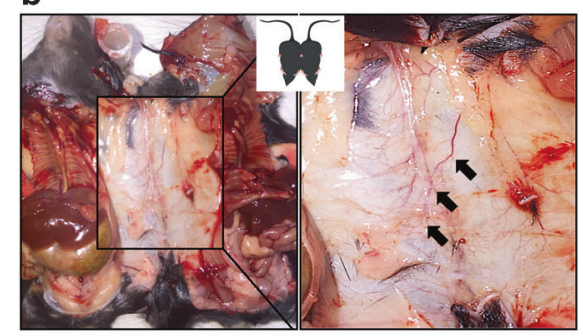

d

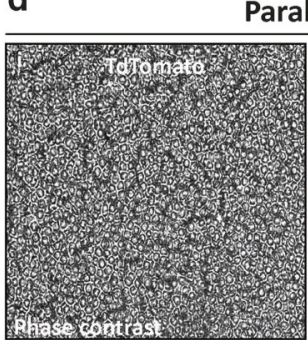

Parabiosis
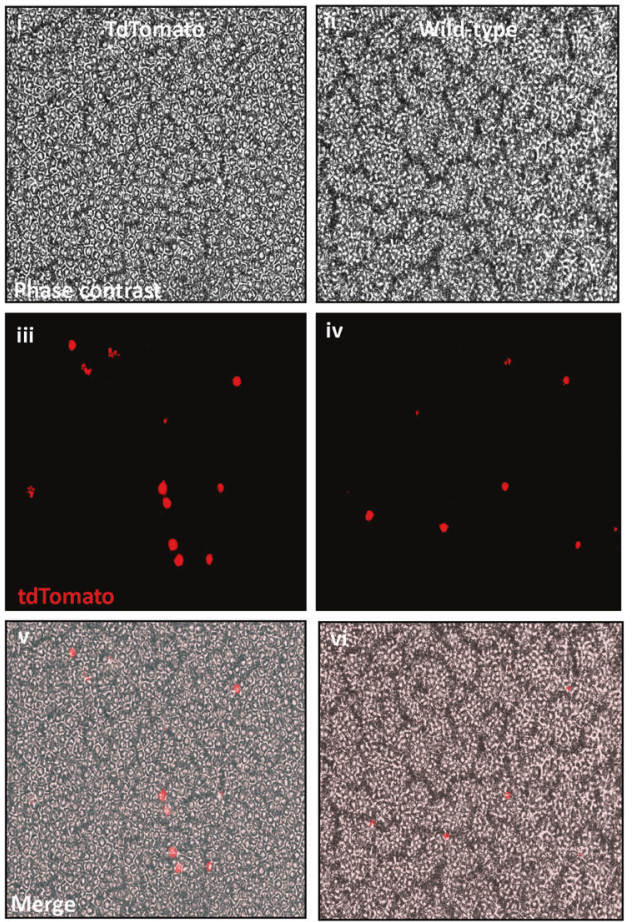

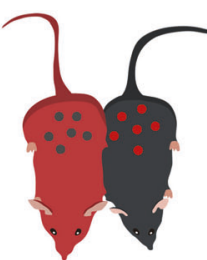

3 days

Radiation or

TNBS injury

to wild-type

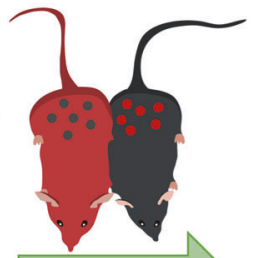

Healing factor

C

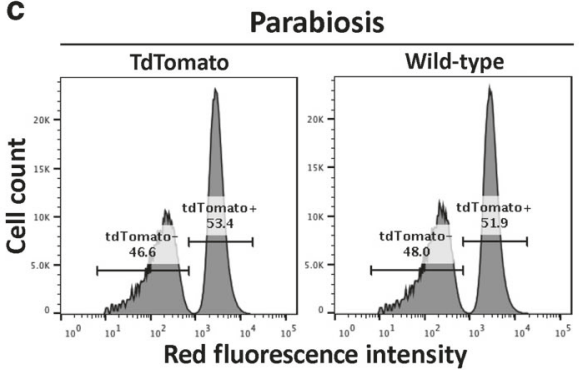

e

Parabiosis
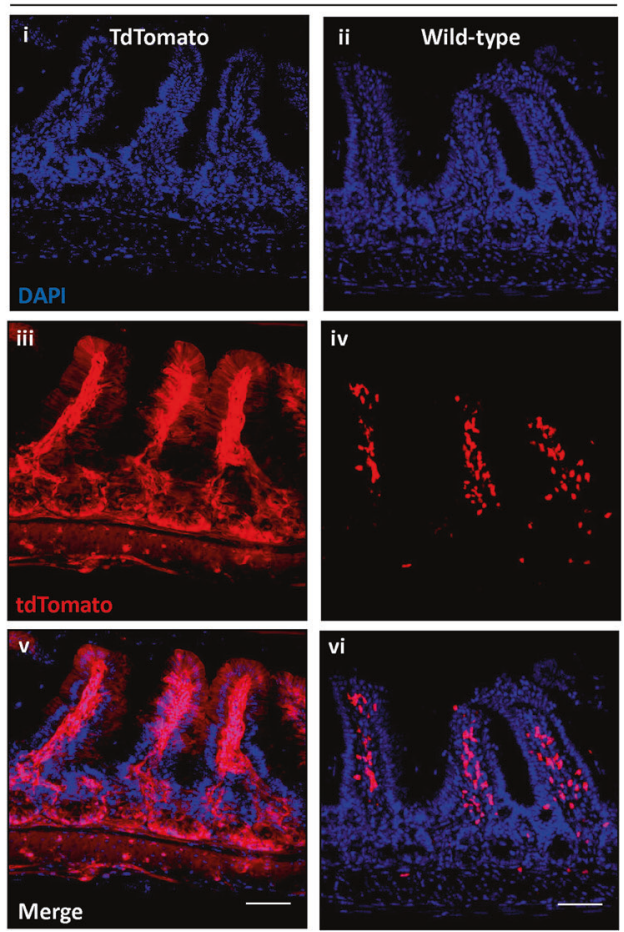

Fig. 1 Establishment of a parabiosis system in mice. a Diagram illustrating the murine parabiosis system. b Blood vessels (arrows) developed across the suture site where the skin of two animals was conjoined. c Flow cytometry analysis revealing that $50 \%$ of tdTomato ${ }^{+}$nucleated blood cells in the peripheral blood of a wild-type parabiont ( $n=4$ pairs) conjoined to a tdTomato mouse. $\mathbf{d}$ Blood smear showing the presence of tdTomato ${ }^{+}$blood cells in a tdTomato mouse (i, iii, and v) and a wild-type mouse (ii, iv, and vi). Original magnification $\times 20$. e Microscopic analysis of the small intestine of a tdTomato mouse conjoined with a wild-type mouse, in which tdTomato ${ }^{+}$cells were observed in the small intestine of a tdTomato mouse (i, iii, and v) and a wild-type mouse (ii, iv, and vi). Original magnification $\times 20$; scale bars $100 \mu \mathrm{m}$

To evaluate the degree to which a shared circulation developed, we observed the macroscopic appearance of bridging blood vessels across the incision between the paired mice (Fig. 1b). Flow cytometric analysis of wild-type-to-tdTomato pairings revealed that the blood of wild-type mice contained $\sim 52 \%$ tdTomato $^{+}$labeled cells (Fig. 1c), which could also be readily appreciated on the blood smear (Fig. 1d). To determine the extent of chimerism in the setting of parabiosis, we paired mice that expressed GFP on all cells under the CAG promoter (so-called ROSA26-CAG-GFP mice) with the ROSA26-CAG-tdTomato mice above, and measured the number of green cells in the lamina propria in the tdTomato mice and the number of red cells in the lamina propria of the GFP mice. As shown in Supplemental Fig. 1, the number of GFP ${ }^{+}$cells that were transferred into the tdTomato recipient was equal to the number of tdTomato ${ }^{+}$cells that were transferred into the GFP mice, indicating that chimerism had been established. In wild-type mice that were paired with tdTomato mice, we detected tdTomato $^{+}$-labeled cells in the lamina propria of the wild-type 
$a_{i}$

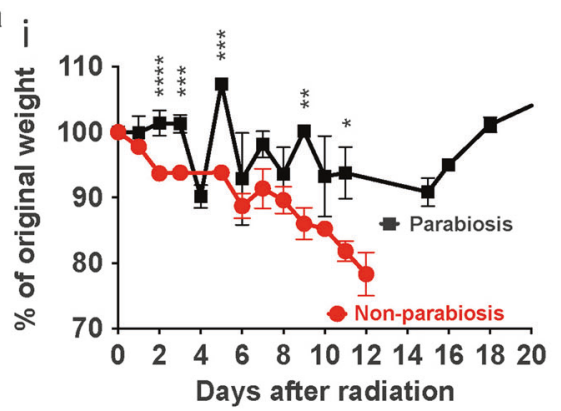

b

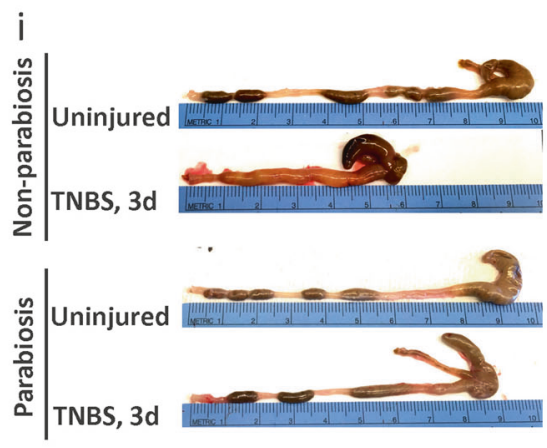

c

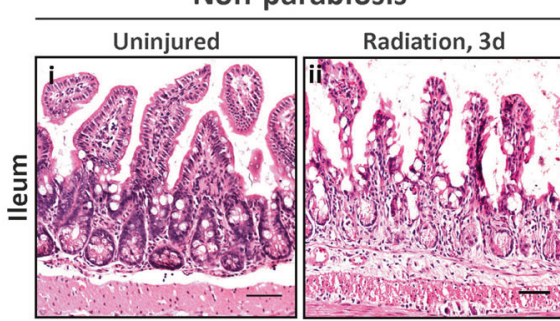

d

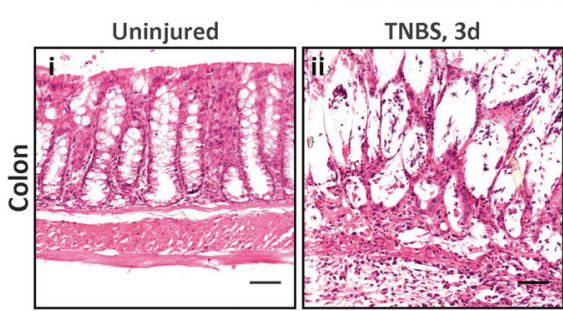

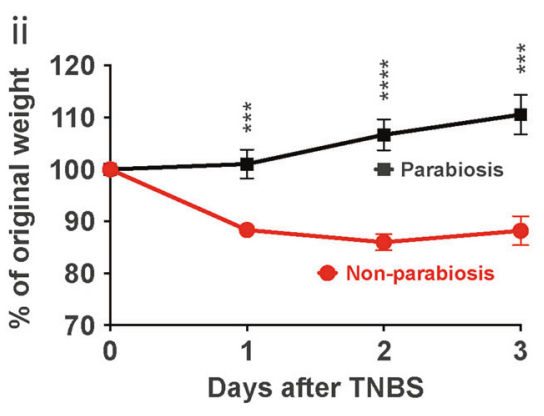

ii

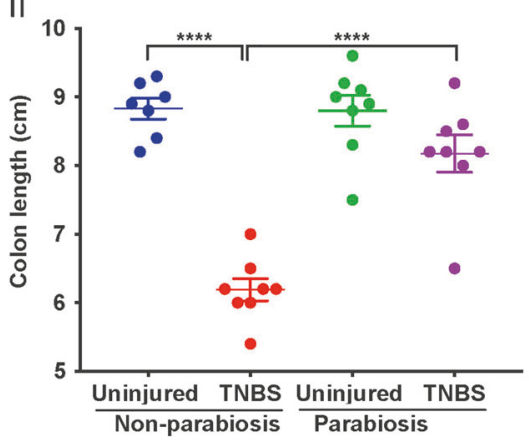

Parabiosis
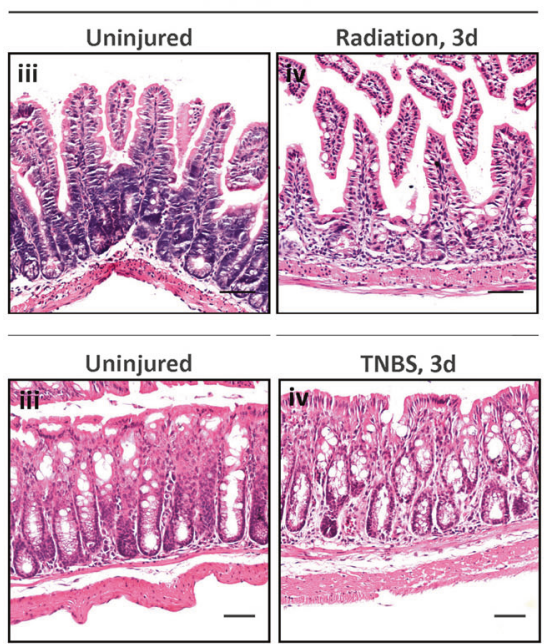

ii

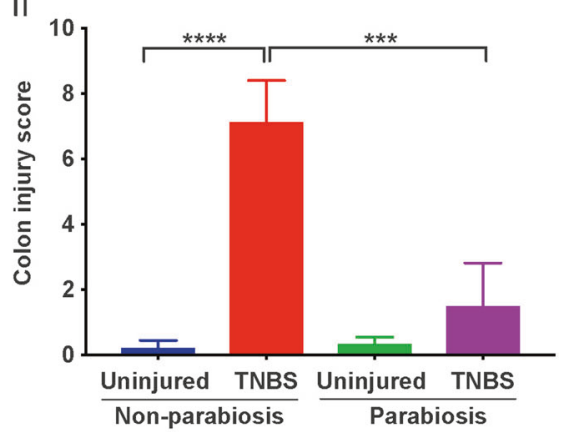

The presence of an uninjured parabiotic partner protects mice against intestinal injury

The effect of parabiotic pairing on gastrointestinal mucosal injury was assessed using two complementary models, namely radiation mice (Fig. 1e), which therefore provided us with an opportunity to determine whether circulating cells from the donor mouse could influence intestinal healing after mucosal injury, as will be evaluated below. 
Fig. 2 Mice with a parabiotic partner are protected from radiation-induced enteritis and TNBS-induced colitis. a (i) Body weight change in an unpaired mouse $(n=14)$ and parabiont mouse $(n=7$ pairs) after exposure of $8 \mathrm{~Gy}$ radiation. (ii) Body weight change in an unpaired mouse $(n=13)$ and parabiont mouse ( $n=11$ pairs) after $2.5 \mathrm{mg}$ TNBS instillation. Mice whose weight loss of $20 \%$ or more were euthanized. Data are presented as means \pm SEM. b (i) Macroscopic images of colons from day 3 after $2.5 \mathrm{mg}$ TNBS instillation. (ii) Colon length of $2.5 \mathrm{mg}$ TNBStreated unpaired mice $(n=7-8$ as indicated) and parabiosis mice $(n=8$ pairs). Data are presented as means \pm SEM. c Representative H\&Estained sections from an unpaired mouse ( $\mathrm{i}$ and ii) and parabiont mouse (iii and iv). lleum 3 days after exposure of $12 \mathrm{~Gy}$ radiation. d Representative H\&E-stained sections from an unpaired mouse (i and ii) and parabiont mouse (iii and iv). Colon 3 days after 2.5 mg TNBS instillation. Original magnification $\times 20$; scale bars $100 \mu \mathrm{m}$. e Histological score by a pathologist blinded to the groups. (i) An unpaired mouse ( $n=5-6$ as indicated) and a parabiont mouse ( $n=3$ pairs) from 12 Gy radiation injury model. (ii) An unpaired mouse ( $n=8-9$ as indicated) and a parabiont mouse ( $n=6$ pairs) from 2.5 mg TNBS injury model. Data are presented as means \pm SEM $\left({ }^{*} p<0.05 ;{ }^{* *} p<0.01 ;{ }^{* *} p<0.001\right.$; $* * * * p<0.0001)$

enteritis-which damages the intestinal stem cells, ${ }^{21}$ and $2,4,6-$ trinitrobenzene sulfonic acid (TNBS)-induced colitis-which causes direct injury of the colonic mucosa. ${ }^{22,23}$ As shown in Fig. 2ai, when unpaired mice were exposed to $8 \mathrm{~Gy}$-irradiation injury, they experienced significant weight loss, and were euthanized 12 days after the radiation exposure because their weights dropped to below $80 \%$ of the original body weight. Strikingly, the body weight of mice who were attached to a parabiosis partner and who were irradiated at the same dosage did not lose weight, and were found to be at their original body weight by day 12 (Fig. 2ai). In the unpaired radiated mice, histological evaluation at 3 days post-irradiation revealed a reduction in the numbers of crypts and atrophic villi accompanied by the infiltration of inflammatory cells into the lamina propria (Fig. $2 \mathrm{c}$ and ei), while the presence of a parabiotic pairing significantly reduced the degree of histologic evidence of crypt-villus injury after radiation (Fig. 2c and ei).

To evaluate whether the protection against intestinal mucosal injury provided by a parabiotic pairing was specific to radiation enteritis, we repeated the above experiments in a model of TNBS colitis. Unpaired mice with TNBS colitis sustained significant weight loss which was most pronounced on day 3 after TNBS instillation (Fig. 2aii), as well as macroscopic evidence of colitis including a shortened, thickened colon (Fig. 2b), and histologic evidence of epithelial injury, crypt degeneration, and inflammatory infiltrate into the lamina propria (Fig. $2 \mathrm{~d}$ and eii). Importantly, these changes were all significantly attenuated in mice with a parabiotic partner (Fig. 2d and eii). Interestingly, the surgical separation of parabiosis pairs $24 \mathrm{~h}$ prior to TNBS exposure partially reversed the rescue effects (Supplementary Figure 2), as observed in body weight changes, gross morphology and histology of colon, indicating that the continuous delivery of cells from the parabiotic partner is required for the augmented healing response. The induction of both radiation-and TNBS-intestinal injury caused an increase in the gene expression of proinflammatory cytokines and chemokines in the intestinal mucosa of unpaired mice (Fig. 3a), as well as an increase in apoptosis of epithelial cells along the crypt-villus axis (Fig. 3b, c), which are known findings in the setting of mucosal injury. ${ }^{24-26}$ By contrast, the presence of a parabiotic partner significantly reduced the expression of pro-inflammatory cytokines and the extent of crypt apoptosis in the injured mouse, further proof of the reduction in intestinal injury that occurs in the presence of parabiosis. Furthermore, the presence of a parabiotic partner reversed the negative effects of radiation injury and TNBS on crypt proliferation (Fig. 3d, e), while TNBS colitis was associated with crypt derangement in the colon in unpaired mice, which was restored in the presence of a parabiotic pairing. Taken together, these findings illustrate the protective effects of a parabiotic partner on mucosal healing in the small and large intestine after epithelial damage.

Based upon the above findings, we next sought to further characterize the protective ability of parabiotic pairing against intestinal injury. As shown in Fig. 4, either TNBS or radiation exposure caused significant disruption in the molecular architecture of the intestinal epithelium, characterized by a reduction in expression of differentiated epithelial markers sucrose isomaltase (SI), MUCIl, and lysozyme, Chromogranin A, CAR1, and Lgr5, which were each restored in the presence of a parabiotic partner (Fig. 4a). Further, the expression of the goblet cell marker Alcian blue and the epithelial marker epithelial-cadherin (Ecad) were both reduced in the presence of TNBS and radiation injury, consistent with prior reports, ${ }^{27-29}$ yet the expression of both of these cellular makers was restored in the presence of parabiosis (Fig. 4b-e). These findings illustrate that parabiotic pairing significantly attenuates intestinal mucosal injury in two different experimental models. We therefore next sought to evaluate the mechanisms involved, and focused first on evaluating whether there was a requirement for circulating cellular versus non-cellular factors from the uninjured donor mouse that could promote healing of the damaged mucosa.

Extra-intestinal cells home to the intestine of the parabiotic pair after intestinal injury

We next evaluated whether extra-intestinal cells of bone marrow origin could migrate from an uninjured mouse to the intestine of an injured mouse and reduce the degree of mucosal injury. To first evaluate the possibility that bone marrow-derived cells could migrate to sites of mucosal injury, we harvested bone marrow cells from an unperturbed tdTomato mouse and injected these cells directly into an unpaired recipient which had undergone radiation injury (12 Gy) $24 \mathrm{~h}$ earlier. As shown in Fig. $5 \mathrm{a}$, we could detect tdTomato $^{+}$cells in the recipient's small intestine 5 days after injection. Having shown that bone marrow-derived cells could home to injury sites, we next investigated whether cells from uninjured parabiotic tdTomato mice could migrate to the injured intestine of wild-type parabionts, and thus improve mucosal healing as shown above. In both radiation enteritis and TNBS colitis (Fig. 5a), numerous tdTomato ${ }^{+}$cells were detected in the lamina propria of the injured wild-type parabiont, indicating that cells migrated from the uninjured donor partner into the injured gut (Fig. 5a). Interestingly, in non-injured parabiosis controls, tdTomato $^{+}$cells were also observed in the lamina propria of the wild-type recipient despite the lack of intestinal inflammation in either mouse of the pair, a finding which is consistent with a shared circulating cell population in the parabiosis system (Fig. 1e). In all cases, only $\sim 2 \%$ of tdTomato-positive cells co-localized with the epithelial marker Ecad (Table 1), suggesting that the migrating cells do not acquire epithelial markers, and thus likely do not become incorporated into the epithelium directly. The tdTomato ${ }^{+}$ cells that were incorporated in the lamina propria did not colocalize with $\mathrm{gp} 8^{+}$(a lymphatic endothelial cell marker) cells, aSMA (a mesenchymal stromal cell marker), and CD45 (a hematopoietic lineage cell marker) (Fig. 6a-c), confirming their non-hematopoietic origin. To further characterize the cells that migrate to the lamina propria of the injured mice, we therefore focused on cells that express the non-hematopoietic markers Sca1, c-kit, and CXCR4, which were expressed by cells in the bone marrow of tdTomato donor parabionts (Fig. 6di-iii) and on the tdTomato $^{+} \mathrm{CD}^{-} 5^{-}$cells, which were recruited to the lamina propria of the injured wild-type recipients (Fig. 6div-vi). These 
a $\mathbf{i}$
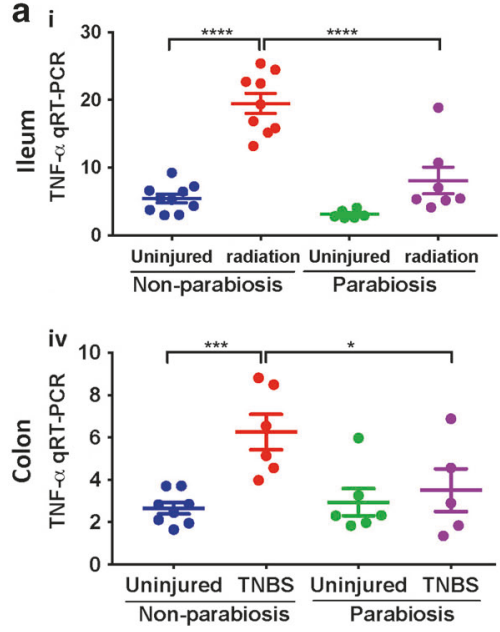

b

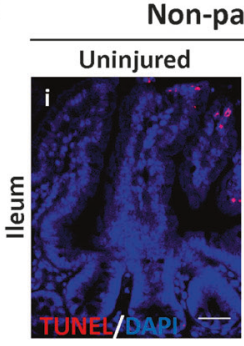

c

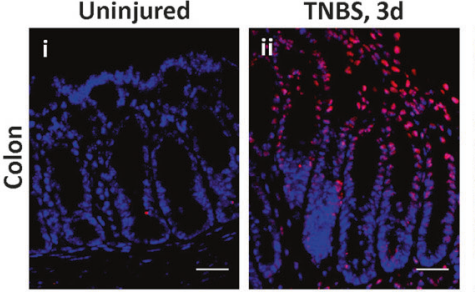

d

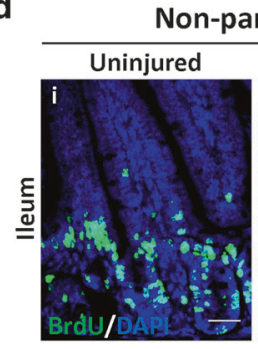

e

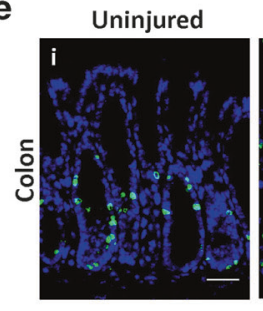

ii
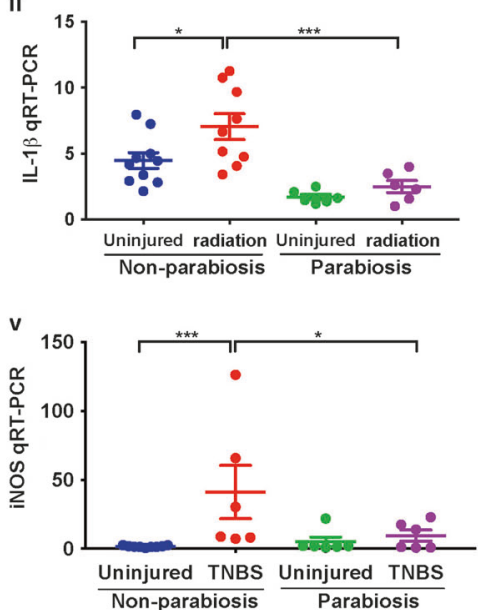

Parabiosis

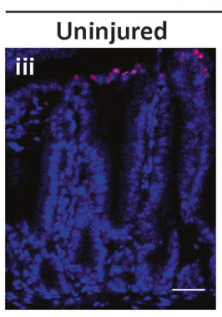

Uninjured

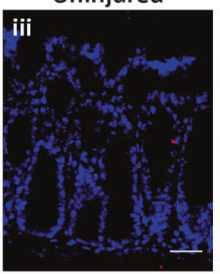

Parabiosis

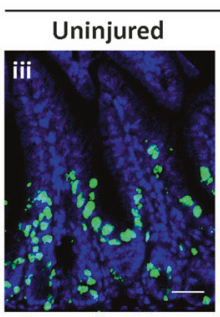

TNBS, 3d
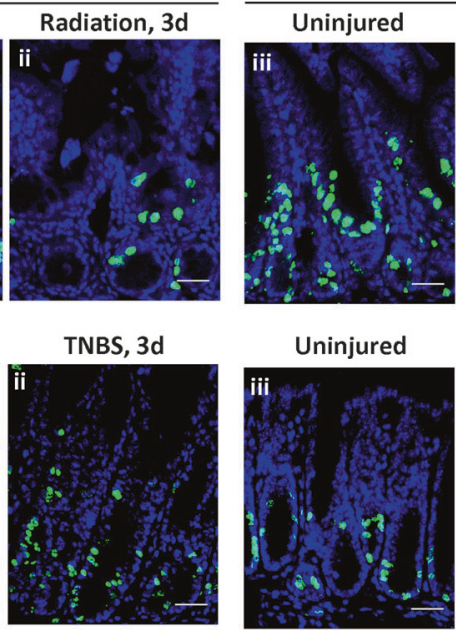
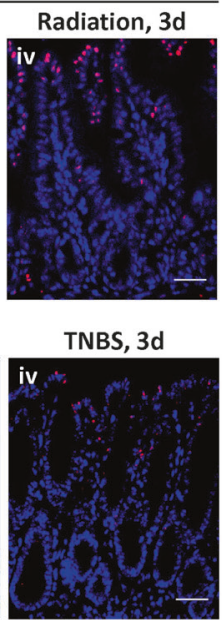

TNBS, 3d

Radiation, 3d

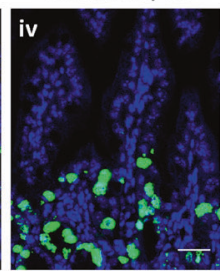

TNBS, 3d

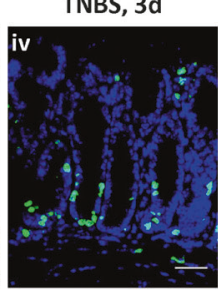

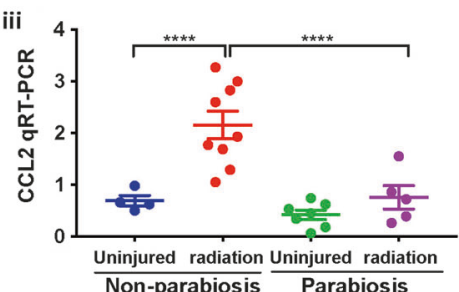
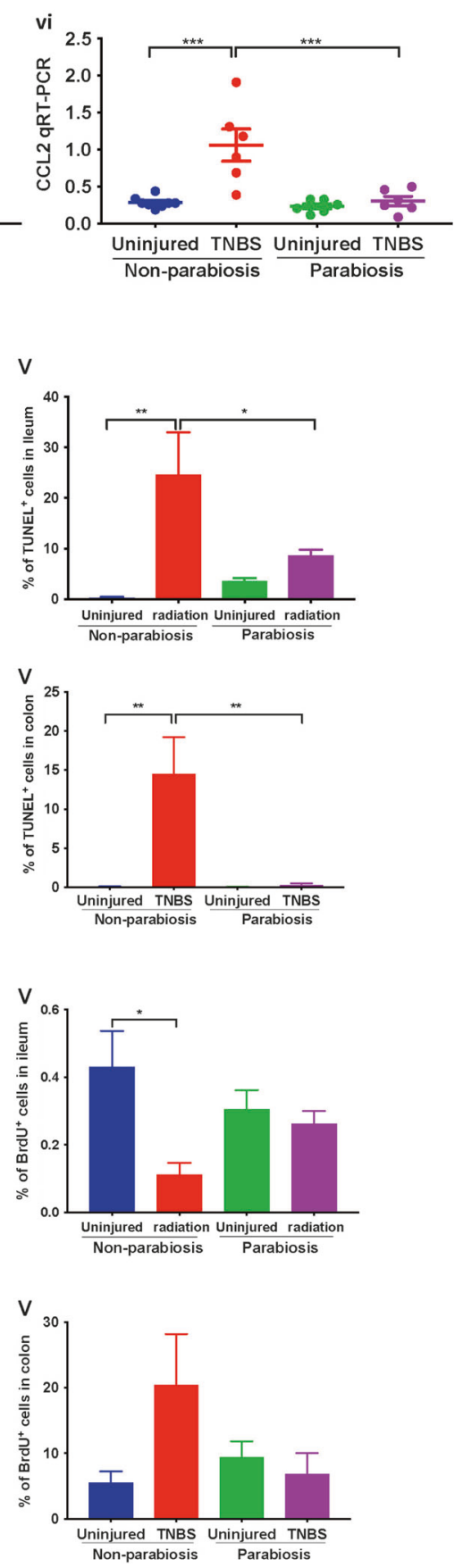

findings indicate that non-hematopoietic cells promote the intestinal healing that is contributed by the presence of a parabiotic partner. We therefore next sought to investigate the functional consequences of the extra-intestinal cells that migrated to sites of mucosal injury in parabiotic mice.
Circulating cells congregate near the crypts of mice subjected to intestinal injury

We next evaluated the distribution of the cells which migrated into the injured mucosa of mice from parabiotic donor. As shown in Fig. 7a, Swiss roll sections and high magnification regions of 
Fig. 3 The presence of a parabiotic partner reduces mucosal injury and improves mucosal healing in the intestine. a RT-qPCR of cytokines and chemokines in the radiation (i-iii) and TNBS (iv-vi) injury models; radiation injury refers to the 8 Gy radiation and TNBS injury refers to the $2.5 \mathrm{mg}$ TNBS instillation as described in Methods; $\mathbf{b}$ Immunohistochemistry of TUNEL (red) and DAPI (blue) in the model indicated; shown (i-iv) is the $12 \mathrm{~Gy}$ radiation injury model. Original magnification $\times 40$; scale bars $50 \mu \mathrm{m}$. (v) Quantification of TUNEL fluorescence intensity normalized to DAPI per $\times 40$ field of view. Each value represents the average of technical replicates from one mouse. c Immunohistochemistry of TUNEL (red) and DAPI (blue) as indicated; shown (i-iv) is the $2.5 \mathrm{mg}$ TNBS injury model. Original magnification $\times 40$; scale bars $50 \mu \mathrm{m}$. (v) Quantification of TUNEL fluorescence intensity normalized to DAPI per $\times 40$ field of view. d Confocal images showing BrdU (green) and DAPI (blue) from $12 \mathrm{~Gy}$ radiation injury model as indicated (i-iv). Original magnification $\times 40$; scale bars $50 \mu \mathrm{m}$. (v) Quantification of BrdU fluorescence intensity normalized to DAPI per $\times 40$ field of view. e Confocal images showing BrdU (green) and DAPI (blue) from 2.5 mg TNBS injury model as indicated (i-iv). Original magnification $\times 40$ scale bars $50 \mu \mathrm{m}$. (v) Quantification of BrdU fluorescence intensity normalized to DAPI per $\times 40$ field of view. Results are presented as means \pm SEM $\left({ }^{*} p<0.05 ;{ }^{* *} p<0.01 ;{ }^{* *} p<0.001 ;{ }^{* * *} p<0.0001\right)$

uninjured parabiosis pairs revealed that tdTomato $^{+}$cells were detected throughout the lamina propria quite evenly. By contrast, in parabiotic mice subjected to radiation injury, the migrated tdTomato ${ }^{+}$cells were found to be clustered around the damaged crypts (Fig. 7a), suggesting a role in the healing response. Although there was no difference in the total number of tdTomato $^{+}$cells that migrated into injured versus uninjured intestinal mucosa (Fig. 7b), the distribution of these cells was very different. Specifically, when we designated the upper part of the villi as Zone 1 and the lower part, in which proliferating intestinal stem cells reside, as Zone 2 (Fig. 7ci), we determined that significantly more tdTomato ${ }^{+}$cells were detected in Zone 2 in an irradiated parabiont as compared with an uninjured parabiont (Fig. 7cii).

In an important control, the pre-treatment of the tdTomato mice with $12 \mathrm{~Gy}$ radiation significantly reduced the accumulation of tdTomato ${ }^{+}$cells in the injured recipient, strongly suggesting their bone marrow origin (Fig. 7d). We thus finally sought to examine the functional consequences of the cells that migrated into the injured mice from the bone marrow of the parabiotic donor.

Ablation of the bone marrow of the donor mouse reverses the protective effects of the parabiotic partner in models of intestinal injury

In the final series of studies, we sought to determine whether the cells that migrated from the parabiotic partner to the injured mouse intestine were required for the improved mucosal healing observed in the parabiotic pairings as shown in Fig. 2. To do so, we applied low dose $(4 \mathrm{~Gy})$ whole-body irradiation to the tdTomato parabionts to ablate the bone marrow of the donor partner. Four days later, we subjected its wild-type parabiont partner to TNBS colitis. Ablation of the bone marrow by $4 \mathrm{~Gy}$ dosage was confirmed by flow cytometry, which revealed that 4 days after radiation, the fraction of total bone marrow cells and number of CD45 ${ }^{-}$cells were significantly reduced as compared with the untreated mice (Supplementary Figure 3a). As shown in Fig. 8a-e, the ablation of the bone marrow from the uninjured donor removed the protective effects of the parabiotic pairing on TNBSinduced weight loss (Fig. 8a), colon length (Fig. 8b), histologic evidence of epithelial injury (Fig. $8 c$ ), the expression of proinflammatory cytokines (Fig. 8d), degree of apoptosis, and proliferation (Fig. 8e) as compared with the parabiotic parings in which the donor mouse had an intact bone marrow.

One possible explanation for the above findings is that adaptive immune cells may play a role in the healing response that is achieved in the presence of parabiosis. To test this possibility directly, we paired the Rag $1^{-/-}$mouse-which lacks an adaptive immune system-with wild-type mice and induced intestinal injury via radiation as above. As shown in Supplemental Fig. 4, the adaptive immune cells were found to be dispensable for the effect of parabiosis on the reduction of intestinal inflammation after radiation, as a reduction in injury was still observed after administration of radiation in the presence of $\mathrm{Rag}^{-1-}$ to wildtype parabiosis pairings. An alternative explanation of the observed rescue by a parabiotic partner is that the rescue may be attributable to a higher quantity of bone marrow cells in the parabiosis system. Interestingly, injection of bone marrow cells into unpaired TNBS-treated mice did achieve some rescue (Supplemental Fig. 5a-c), although to a lower degree than that which was observed in the parabiotic pairings under the same condition of TNBS administration. In additional studies, we injected $\mathrm{CD}_{4} 5^{-}$and $\mathrm{CD} 45^{+}$bone marrow cells into unpaired radiated mice, and determined that $\mathrm{CD}_{4} 5^{-}$bone marrow cell injections achieved greater gastrointestinal healing as compared to $\mathrm{CD} 45^{+}$bone marrow cell injection (Supplementary Figure $5 \mathrm{~d}$ and e), providing additional details that it is the $\mathrm{CD}^{-} 5^{-}$bone marrow cells that mediate the healing effect. Finally, in an attempt to pinpoint the homing pathways by which the bone marrowderived cells migrate to the sites of mucosal inflammation, we examined the role of two different homing mechanisms-C-C chemokine receptor type 2 (CCR2) and mucosal addressin cell adhesion molecule-1 (MAdCam-1) - in the acute colitis injury model. While inhibition of CCR2 did not affect the protective effects of the parabiosis pairing, the inhibition of MAdCam-1 reversed the previously observed rescue effects in the parabiosis system (Supplementary Figure 6), indicating the involvement of MAdCam-1 in the homing of the circulating cells to the injured intestine. Taken together, these findings illustrate that a parabiotic partner delivers bone marrow-derived cells which then reduce the extent of intestinal injury in mice, and suggest that extra-intestinal cells contribute to the healing response.

\section{DISCUSSION}

The current study reveals that in two models of intestinal injury, the degree of epithelial damage can be significantly reduced if the injured mouse is surgically connected to a parabiotic partner. In seeking to define the mechanisms involved, we demonstrate that circulating cells from a parabiotic partner can migrate to the sites of mucosal injury, where they then reduce the degree of injury and enhance mucosal healing. In assessing the mechanisms of protection, migrated cells were congregated near the proliferating crypts, where they contribute to the healing response in a paracrine manner. Strikingly, ablation of the bone marrow of the donor partner by low dose irradiation removed the protective effect of the parabiotic pairing on the extent of TNBS colitis, revealing that it is the cells themselves as opposed to a noncellular factor that contributes to the beneficial effects of the parabiotic pairing. Taken together, these findings indicate that intestinal injury leads to the recruitment of extra-intestinal cells likely of bone marrow origin to the damaged mucosa, and that these cells play an important role in reducing the extent of inflammation and promoting the healing response.

While the current findings are derived exclusively from animal studies, it is important to understand how these results could apply to human intestinal disease. Radiation enteritis is a significant complication of the treatment of many abdominal cancers, ${ }^{30,31}$ and can be a limiting factor in the provision of prescribed radiation doses in many patients. ${ }^{32,33}$ Current 
$\mathbf{a}_{\mathrm{i}}$
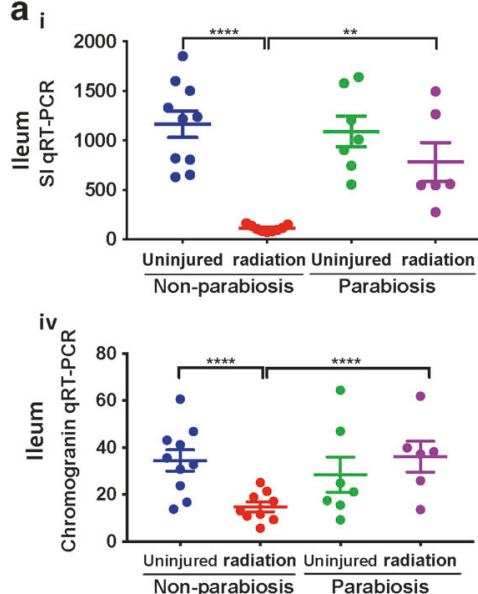

b

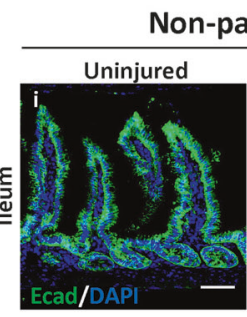

c

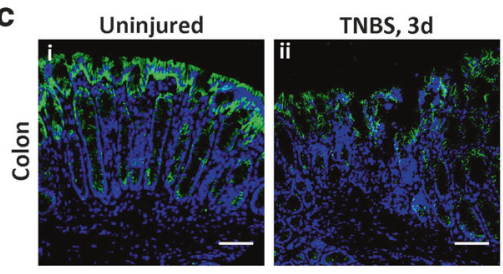

d Non-parabiosis

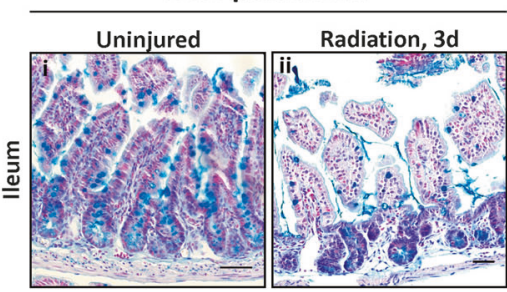

e

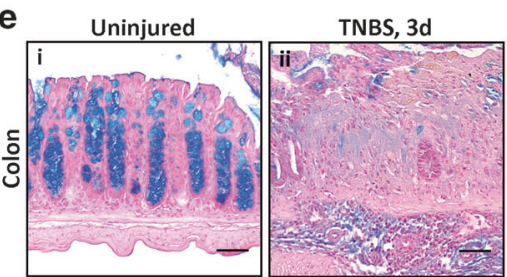

ii

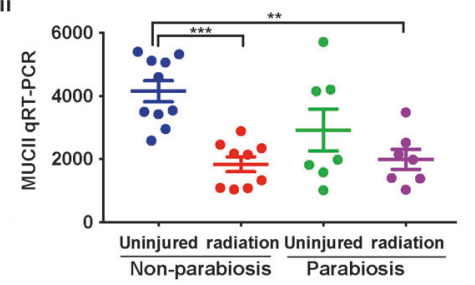

v
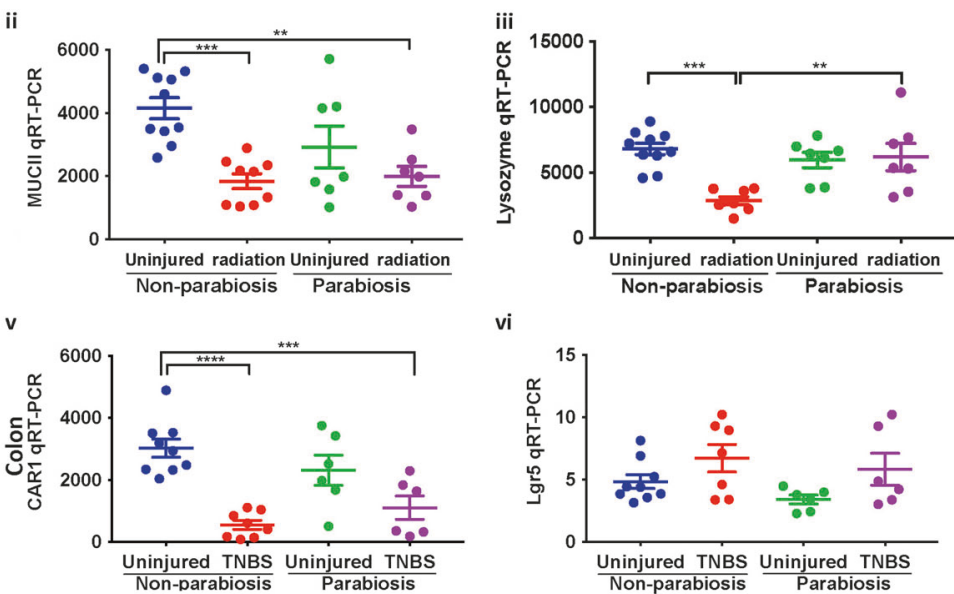

vi

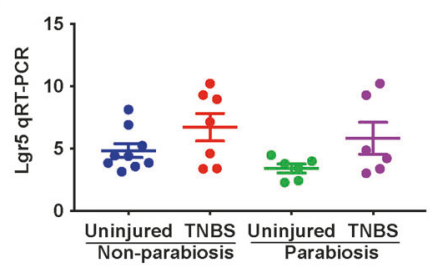

Parabiosis
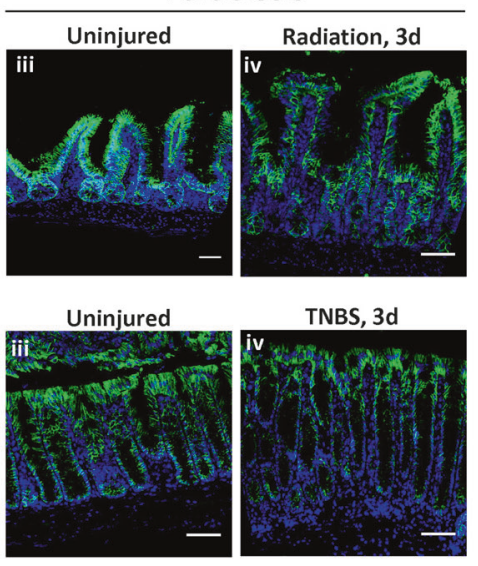

TNBS, 3d

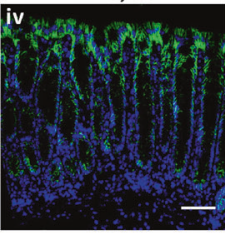

Parabiosis
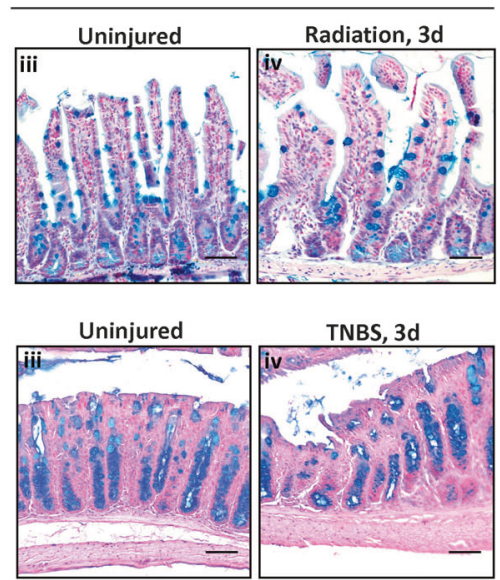

Fig. 4 The presence of a parabiotic partner restores mucosal architecture and epithelial differentiation after injury. a RT-qPCR showing expression of the indicated differentiation markers in the indicated groups in unpaired mice $(n=9-10)$ and parabiont mice $(n=7$ pairs) from 8 Gy radiation injury model (i-iv); colon of unpaired mouse $(n=6-9$ as indicated) and parabiont mouse $(n=7$ pairs) from 2.5 mg TNBS injury model ( $v$ and vi). $\mathbf{b}$ and $\mathbf{c}$ Representative Ecad (green) and DAPI (blue) stained sections from a non-parabiosis mouse (i and ii) and parabiont mouse (iii and iv) as indicated. $\mathbf{d}$ and e Representative Alcian blue-stained sections from a non-parabiosis mouse (i and ii) and parabiont mouse (iii and iv) as indicated; scale bars $100 \mu \mathrm{m}\left({ }^{*} p<0.05 ;{ }^{* *} p<0.01 ;{ }^{* * *} p<0.001 ;{ }^{* * *} p<0.0001\right)$. Data are shown as means $\pm \mathrm{SEM}$

treatment of radiation enteritis is limited to either reducing the total radiation dose that is applied-which can have negative effects on tumor treatment-or attempts to manage the bloody diarrhea and weight loss symptomatically. Similarly, severe cases of inflammatory bowel disease (of which TNBS colitis shares similarities) can be extremely debilitating, resulting in severe pain, weight loss, and death from sepsis in the most severe cases. ${ }^{34,35}$ The finding that extra-intestinal and likely bone marrow-derived cells from a healthy donor can migrate to and attenuate intestinal injury raises the exciting possibility that stem cell transplantation may be utilized in cases of severe inflammation from either radiation enteritis or IBD. In fact, there are case reports showing 

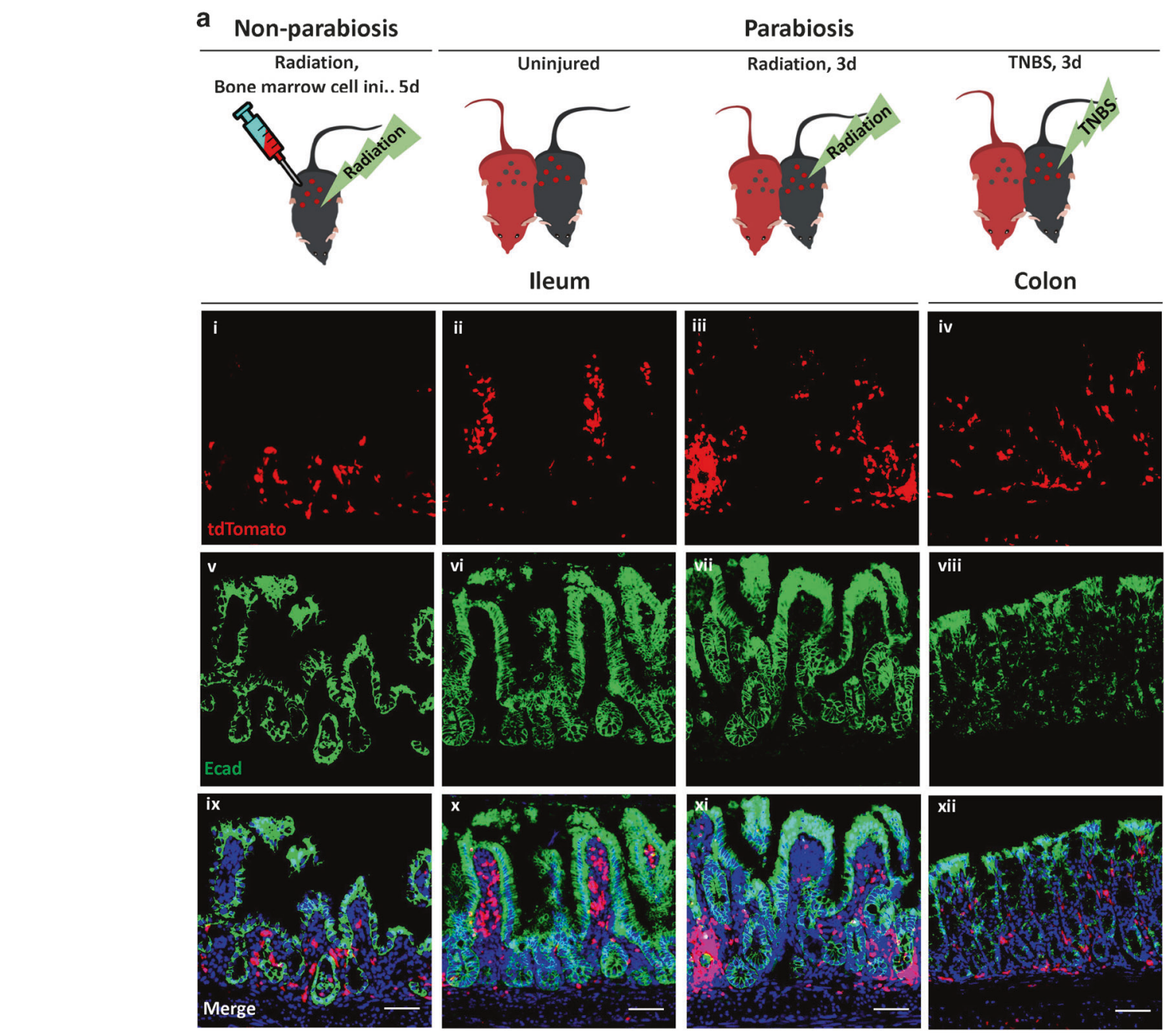

Fig. 5 Injected bone marrow-derived cells and cells of donor parabionts' origin which migrate into injured intestinal mucosa do not colocalize with an intestinal epithelial marker. a Representative images showing the double immunofluorescence of tdTomato and Ecad in bone marrow cell transplant recipients with $12 \mathrm{~Gy}$ radiation (i, v, and ix), uninjured parabionts (ii, vi, and $\mathrm{x}$ ), $8 \mathrm{~Gy}$-radiated parabiosis mice (iii, vii, and $\mathrm{xi}$ ), and $2.5 \mathrm{mg}$ TNBS-treated parabiosis mice (iv, viii, and xii). Original magnification $\times 20$; scale bars $100 \mu \mathrm{m}$

Table 1. Quantification of tdTomato ${ }^{+}$cells that co-localize with the epithelial marker E-cadherin

\begin{tabular}{ll}
\hline Group & $\%$ tdTomato $^{+} \mathrm{Ecad}^{+}$cells \\
\hline Radiation, bone marrow cell inj., non- & 0.3 \\
parabiosis & \\
Uninjured, parabiosis & 2.2 \\
Radiation, parabiosis & 1.5 \\
TNBS, parabiosis & 0.2 \\
\hline
\end{tabular}

The percentage of tdTomato/Ecad-double positive cells out of total tdTomato $^{+}$cells shows the proportion of donor-derived intestinal epithelial cells in bone marrow cell transplant recipients with $12 \mathrm{~Gy}$ radiation $(n=2)$, uninjured parabionts $(n=2$ pairs), 8 Gy-radiated parabiosis mice ( $n=3$ pairs), and TNBS-treated parabiosis mice $(n=2$ pairs)

success of stem cell transplantation for severe Crohn's disease in patients, although the mechanisms of healing in patients are unknown. $^{36}$ The current studies provide an experimental and biological rationale for their further evaluation.
The current set of experiments extend the work of others who have examined the role of bone marrow-derived cells as an alternative source for replacement cells during injury by differentiating into organ-specific cells. ${ }^{37-39}$ It is noteworthy that several studies in this area have shown somewhat inconsistent findings, regarding both the potential of trans-differentiation of exogenous stem cells into intestinal tissue and the functional significance of these cells on tissue remodeling. ${ }^{14,16}$ Rizvi et al. have shown that transplanted bone marrow cells fuse with intestinal stem cell population, suggesting a critical role of cell fusion events in intestinal regeneration. ${ }^{14}$ In contrast, de Jong's group has shown that the fusion event of intestinal epithelial cells with bone marrow-derived cells is so rare that it is dispensable for intestinal repair process. ${ }^{16}$ This contradiction could be explained by inherent limitations of the bone marrow cell transplantation protocols in which a subset of harvested cells is transplanted, and incorporation can be variable. Moreover, considering the extensive length of the small intestine, a relatively rare population of transplanted cells could easily go undetected. In the present study, utilization of a parabiosis model system in combination of a Swiss roll preparation for histological sectioning circumvents the technical limitations of transplantation, and allows for a bird's-eye view of the entire intestinal length. Additional studies are 
a Non-parabiosis

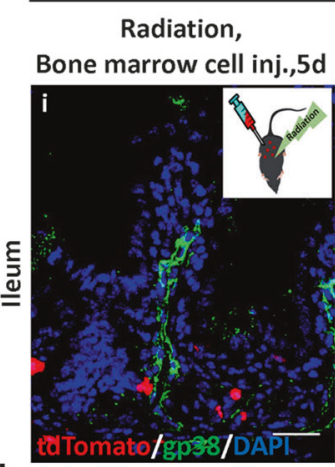

b

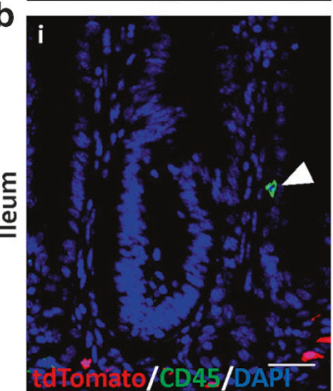

C
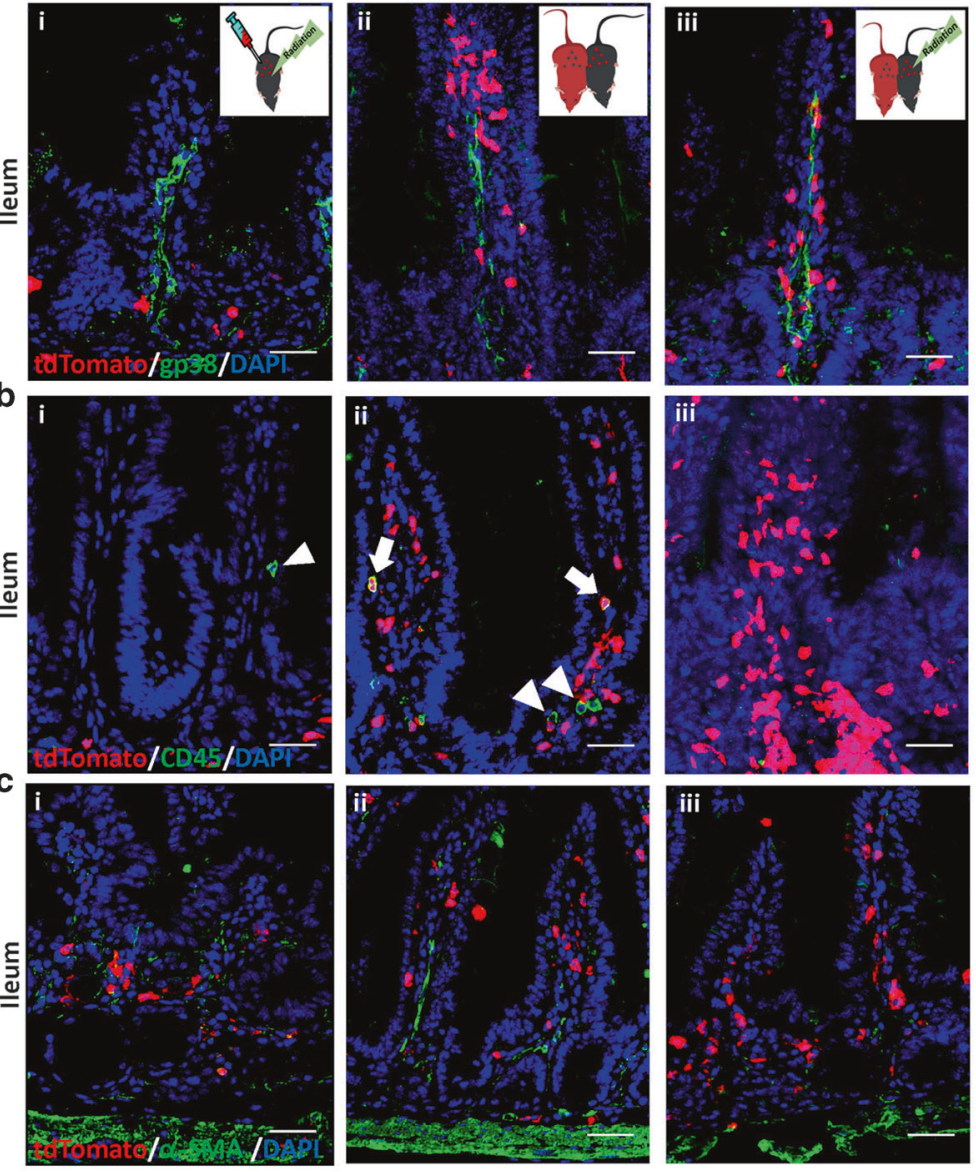

d

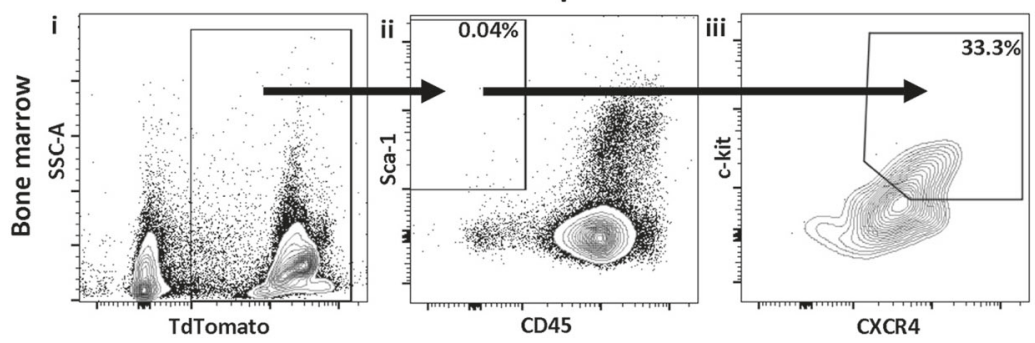

Wild-type parabiont, Radiation, 3d

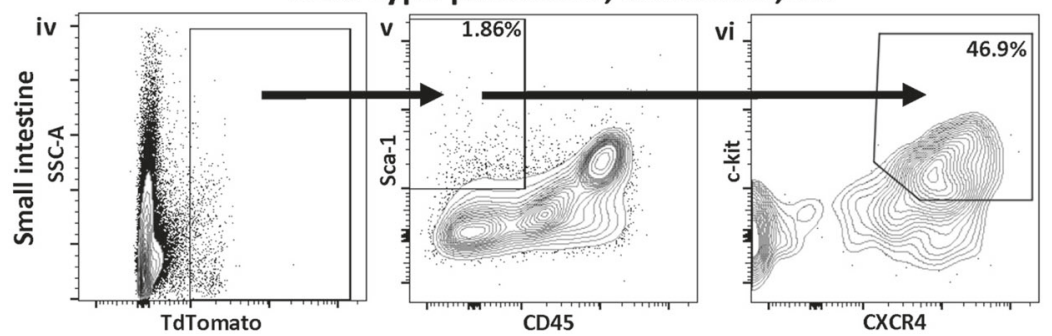

therefore still required to determine whether cell fusion and transdifferentiation can ever occur.

We acknowledge that although the current studies point strongly to the bone marrow as the source of the donor cells in healing from intestinal injury, it is possible that the bone marrow may not be the only source of the cells that migrate to the site of injury, and that some of the healing that is observed in the parabiotic pairing may be attributed to non-cellular pro-healing 
Fig. 6 Analysis of the cells that migrate into the sites of intestinal injury in a parabiosis model. a Representative confocal images showing the immunofluorescence analysis of tdTomato (red) with gp38 (green), (b) tdTomato (red) with CD45 (green) or (c) tdTomato (red) with $\alpha$-SMA (green); from bone marrow cell transplant recipients with 12 Gy radiation (i), uninjured parabionts (ii), and 8 Gy-radiated parabiosis mice (iii). Original magnification $\times 40$; scale bars $50 \mu \mathrm{m}$. In (b), arrows indicate double positive tdTomato ${ }^{+} / \mathrm{CD}_{4} 5^{+}$cells, and arrowheads indicate single positive tdTomato ${ }^{-} / \mathrm{CD} 5^{+}$cells in uninjured parabionts. $\mathbf{d}$ Representative gating strategies of tdTomato ${ }^{+}$cells in bone marrow of tdTomato parabionts $\left(n=3\right.$ pairs). TdTomato-positive cells were gated to select CD45 ${ }^{-} \mathrm{Sca}^{-}{ }^{+}$c-kit $^{+} \mathrm{CXCR}^{+}$cells in bone marrow (i-iii). The abovementioned gating strategies were used to select tdTomato ${ }^{+} \mathrm{CD}_{4} 5^{-} \mathrm{Sca}-1^{+} \mathrm{c}^{-k i t}{ }^{+} \mathrm{CXCR} 4^{+}$cells (iv-vi) in lamina propria of $8 \mathrm{~Gy}$-radiated parabionts ( $n=3$ pairs)

factors. ${ }^{40,41}$ For instance, it has previously been reported that mesenchymal stem cells-which can contribute to intestinal mucosal healing, ${ }^{42}$ can be isolated from not only the bone marrow but also the adipose tissue. ${ }^{40,43}$ Moreover, pro-healing factors from a donor source-including epidermal growth factor (EGF), IL-10, and/or transforming growth factor-beta1 (TGF- $\beta 1$ ) could contribute to the healing response as has been reported previously, $41,44,45$ although the fact that rag-/- mice donors still promote healing minimizes a role for the adaptive immune system in the healing response. Additional studies will be required to delineate these various possibilities, although the fact that ablation of the bone marrow of the donor mouse reversed the previously observed rescue effects argues strongly in favor of a cellular-mediated mechanism of gut protection from the parabiotic donor.

Previous authors have utilized a parabiosis system to elucidate the mechanisms underlying healing from mucosal injury, ${ }^{46-49}$ and we now extend these prior observations. For instance, Tomita, T. et al. have shown that the development of a malabsorptive wasting disease in a parabiosis system in which IL-7 knockout mice were paired with a colitic IL-7 mouse, indicating that systemic IL-7 is necessary for the development and maintenance of colitis. ${ }^{46,47}$ The same group also have shown that the presence of colitogenic $\mathrm{CD}^{+}$effector-memory $\mathrm{T}$ cells in peripheral blood exacerbates the chronic colitis using a parabiotic system. ${ }^{47}$ However, Ono, Y. et al. utilized a parabiosis system to reveal that naturally occurring Th17 cells play a protective role under steady state, while colitogenic Th17 cells contribute to pathogenesis of chronic colitis during inflammatory conditions. ${ }^{48}$ Mikami, Y. et al. have shown that healing of colitis can be achieved in parabiosis mice by the competition between Th1 and Th17 cells, which play a critical role in modulating colitis. ${ }^{49}$ The current study broadens these findings by revealing that bone marrow-derived cells can migrate to the recipient lamina propria even in the steady state, and that ablation of the bone marrow reverses all protective effects in models of radiation injury and colitis, demonstrating the functional relevance of their intestinal homing.

In summary, we have shown that extra-intestinal cells which are likely of bone marrow origin can facilitate intestinal healing using a parabiotic system. The current approach which utilizes two intestinal injury models involving both the small intestine and the colon allows us to be more certain of a general role for circulating cells in the healing response. In aggregate, the current experiments increase our understanding of the healing process of the damaged gut, and shed light on the possible utilization of bone marrow cell transplantation as a possible therapy for intestinal diseases.

\section{METHODS}

Statement of ethics

All animal procedures were conducted in accordance with National Research Council's Guide to the Care and Use of Laboratory Animals, and all of the animal protocols have been approved by the Institutional Animal Care and Use Committee (IACUC) at Johns Hopkins university. All mice were housed under a controlled temperature $\left(25^{\circ} \mathrm{C}\right)$ and light-dark cycle of $12 \mathrm{~h}$.
Establishment of parabiotic mouse pairs

Age matched, adult (3-12 months old) female wild-type (C57BL/6), $\mathrm{Rag}^{1 /-}$ mice (C57BL/ Rag1 KO), transgenic tdTomato mice (C57BL/ CAG-tdTomato), or transgenic GFP mice (C57BL/CAG-GFP) in which all cells express tdTomato or GFP were used to generate parabiotic pairs. All mice were purchased from Jackson Laboratory (Bar Harbor, ME). Mice were co-housed for 2 weeks prior to surgery to reduce stress. For the parabiosis procedure, mice were anesthetized by intramuscular injection with $100 \mathrm{mg} / \mathrm{kg}$ ketamine and $15 \mathrm{mg} / \mathrm{kg}$ xylazine. A skin incision was made on the flank from the elbow to the knee joint, and the tibia and ulna of each animal were sutured together with 4-0 biosyn (Covidien) to join together the forelimbs and hindlimbs. The abdominal walls of each mouse were also sutured together using 5-0 monocryl (Ethicon) to promote the formation of a shared circulation between the two animals. The skin incisions on the flanks were then closed using $7 \mathrm{~mm}$ wound clips. Immediately following surgery, each parabiont was administered sterile normal saline to prevent dehydration, Baytril to minimize infection, and Buprenorphine to manage pain.

After 4 weeks, the presence of a paired circulation was assessed by detection of tdTomato ${ }^{+}$cells in blood and intestine in a wildtype parabiont which had been surgically connected to a tdTomato mouse. Blood from the wild-type pair was obtained via retro-orbital bleeding and the peripheral blood was examined by fluorescence microscopy for the presence of tdTomato ${ }^{+}$cells in the wild-type parabionts and by flow cytometry. Additionally, a shared circulation in intestine was examined by pairing a CAGGFP mouse to a CAG-tdTomato mouse, and assessing the presence of $\mathrm{GFP}^{+}$cells in the lamina propria of the CAGtdTomato mice, and tdTomato $^{+}$cells in the lamina propria of the CAG-GFP mice.

For flow cytometry, red blood cells were first removed by incubation in lysis buffer and washed with FACS buffer (0.5\% BSA in PBS, $2 \mathrm{mM}$ EDTA) three times. Cells were examined on a Accuri C6 flow cytometer and analyzed with FlowJo software. In pairings between wild-type and tdTomato mice, the composition of nucleated cells in peripheral blood was $~ 50 \%$ of tdTomatopositive and $50 \%$ tdTomato-negative, consistent with the development of a shared circulation.

\section{Separation of parabiosis mouse pairs}

One month after the recovery from the parabiosis surgery, mice were surgically separated, as follows: mice were anesthetized as above, and a longitudinal incision on the skin and underlying fascia was made resulting in separation of the attached parabiosis pairs. A suture which binds the forelimbs and hindlimbs was cut to free the joints. The skin incision on the flank was then closed with $7 \mathrm{~mm}$ wound clips. In a control group, unpaired mice were subjected to a sham surgery, which involved creation of a skin incision on the flank, which was then closed with $7 \mathrm{~mm}$ wound clips. Immediately following surgery, each mouse was administered sterile normal saline to prevent dehydration and Buprenorphine to manage pain. Twenty-four hours later, the separated or sham-operated mice were induced to develop intestinal injury, as described below. 
Parabiosis
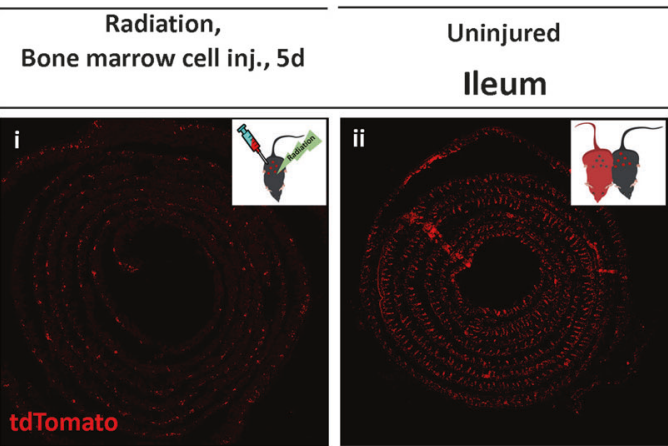

Radiation, 3d
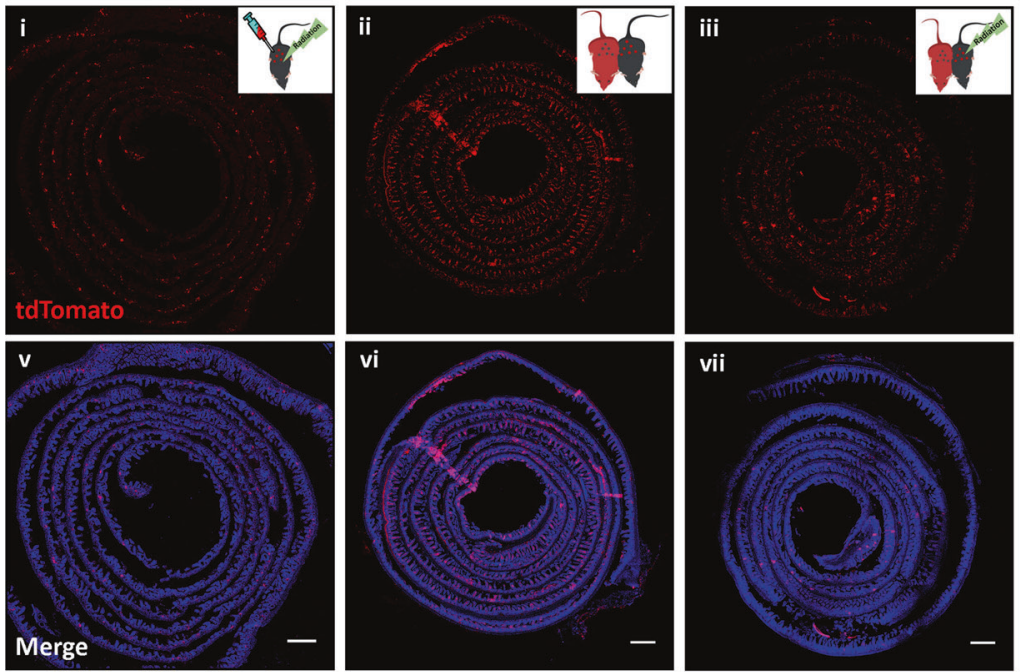

b

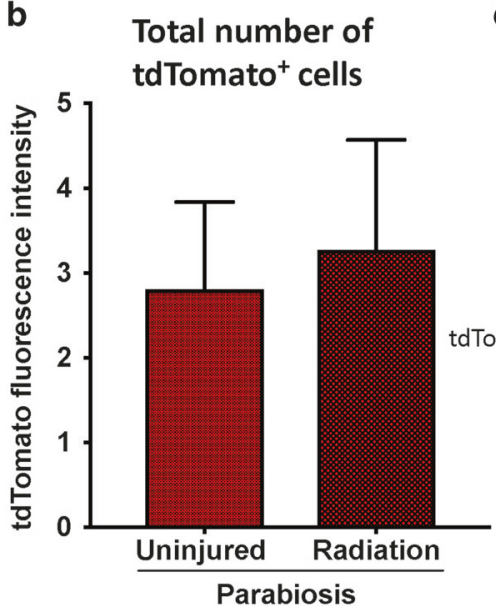

d

c i

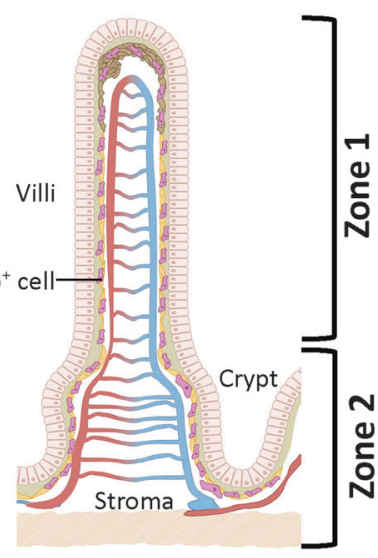

ii TNBS, 3d
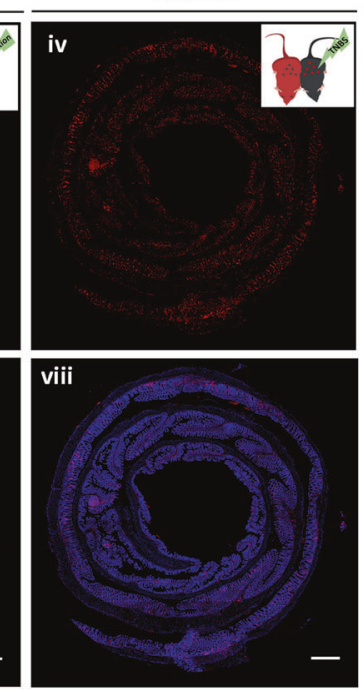

\begin{tabular}{c} 
TNBS, 3d \\
Colon \\
\hline
\end{tabular}

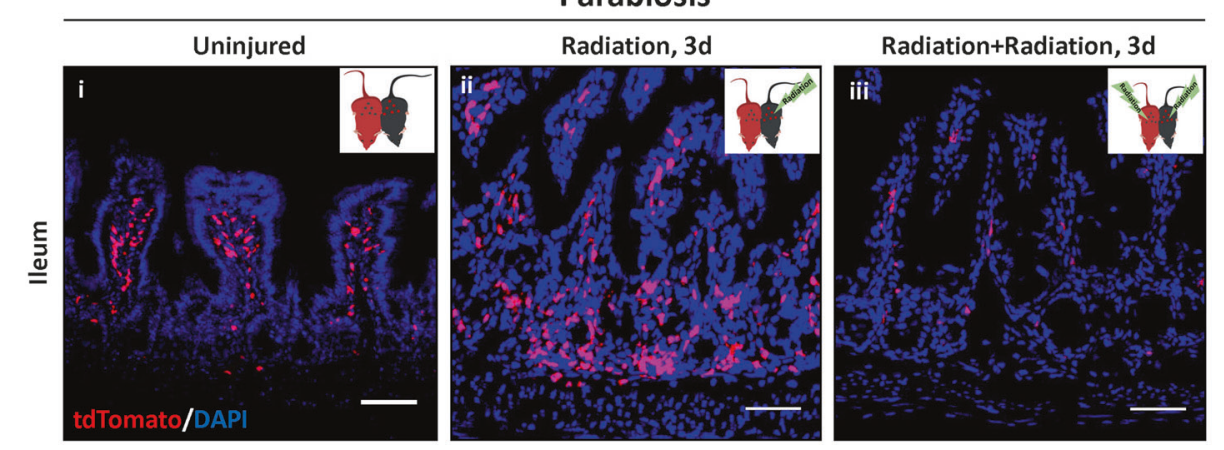

Fig. 7 Extra-intestinal cells congregate near the proliferating crypts and do not accumulate after prior bone marrow depletion. a Immunofluorescence analysis of tdTomato ${ }^{+}$cells in Swiss roll sections from bone marrow cell transplant recipients with $12 \mathrm{~Gy}$ radiation ( $\mathrm{i}$ and v), uninjured parabionts (ii and vi), $8 \mathrm{~Gy}$-radiated parabiosis mice (iii and vii), and $2.5 \mathrm{mg}$ TNBS-treated parabiosis mice (iv and viii). b Quantification of fluorescence intensity of tdTomato ${ }^{+}$cells shows the number of recruited tdTomato ${ }^{+}$cells in small intestine of uninjured parabionts ( $n=3$ pairs) and 8 Gy-radiated parabiosis mice ( $n=3$ pairs). Each value represents fluorescence intensity in $\times 20$ field of view of a single Swiss roll section. Results are presented as means \pm SEM. c (i) The topographical designation of the upper part of villi as Zone 1 and the lower part, in which intestinal stem cells reside, as Zone 2. (ii) Quantification of tdTomato ${ }^{+}$cells depending on their location, Zone 1 versus Zone 2 , in uninjured parabionts ( $n=3$ pairs) and $8 \mathrm{~Gy}$ - radiated parabiosis mice $\left(n=3\right.$ pairs). Results are presented as means \pm SEM $\left({ }^{*} p<0.05\right)$. d Representative images of recruitment of tdTomato ${ }^{+}$cells in the small intestine of uninjured parabionts (i), $12 \mathrm{~Gy}$-radiated parabiosis mice (ii), and $12 \mathrm{~Gy}$ - radiated parabionts whose partners were irradiated with $12 \mathrm{~Gy}$ (iii). Original magnification $\times 20 ;$ scale bars $100 \mu \mathrm{m}$ 

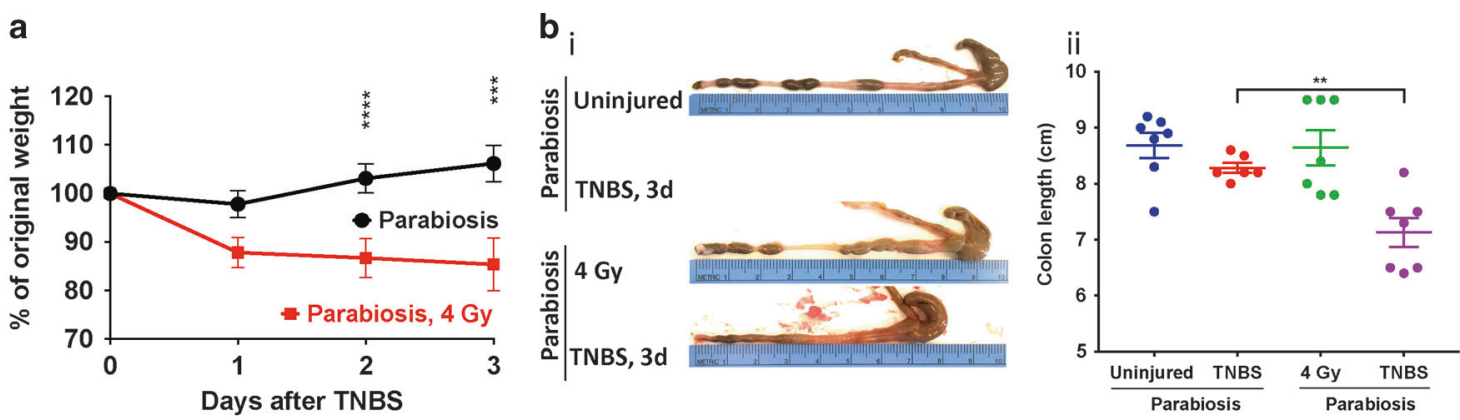

C
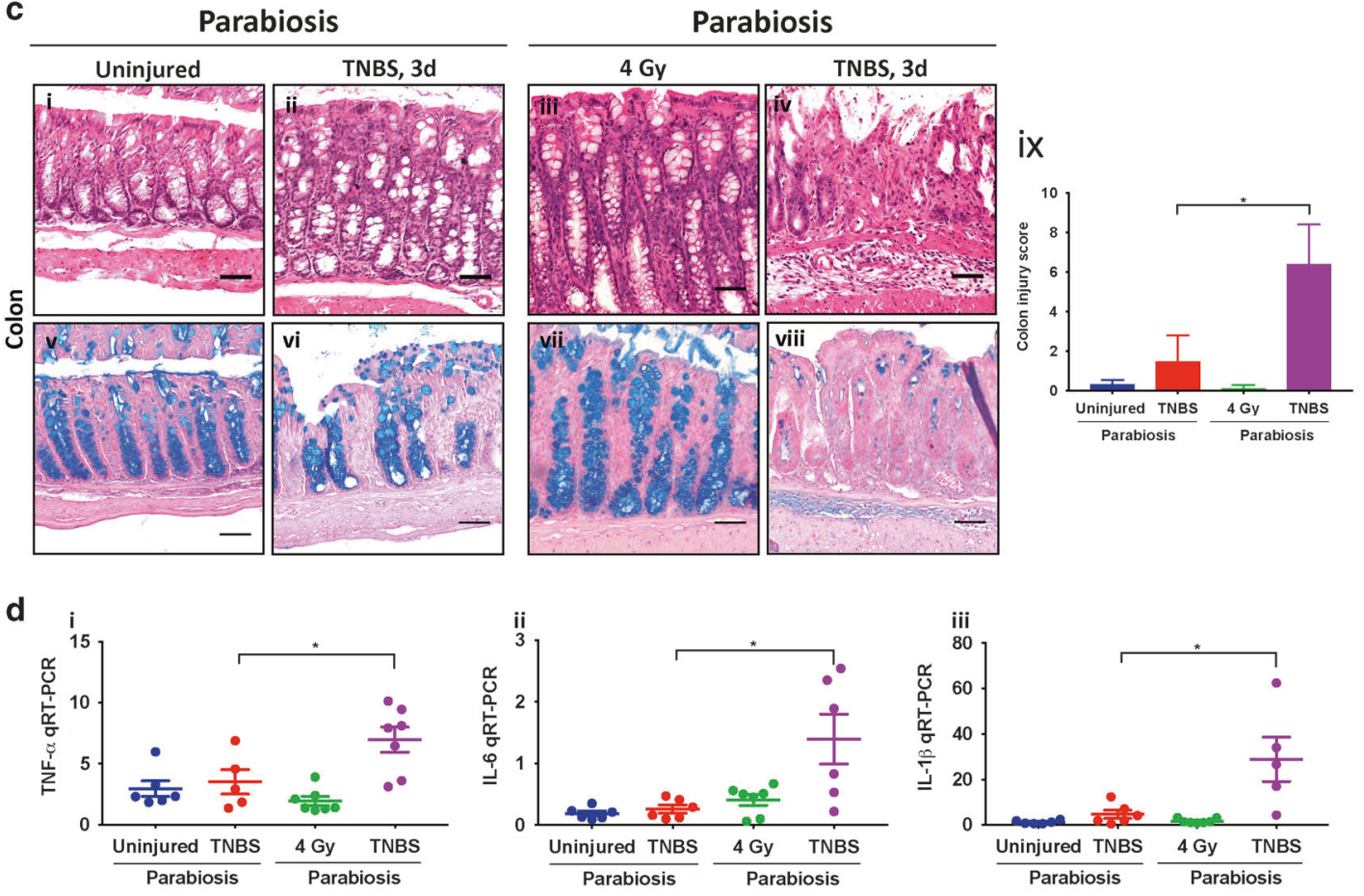

Parabiosis
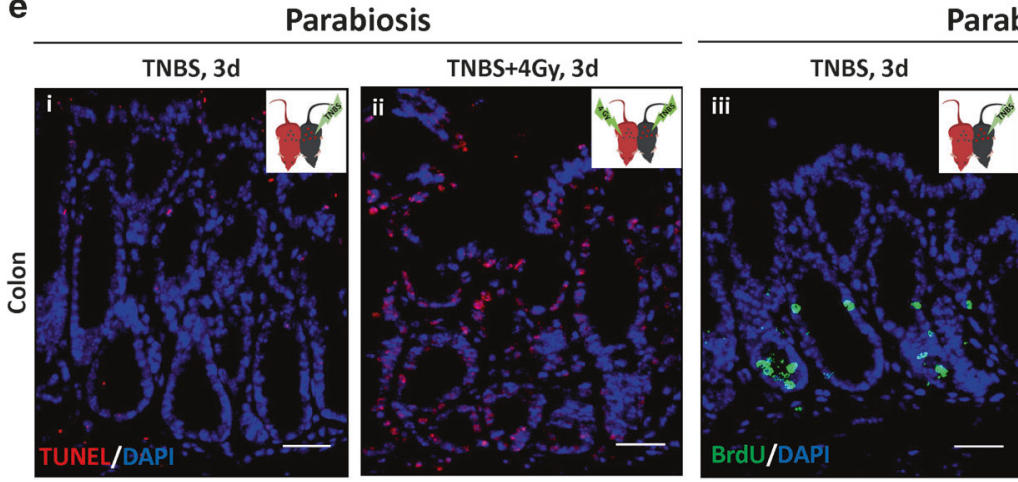

Parabiosis

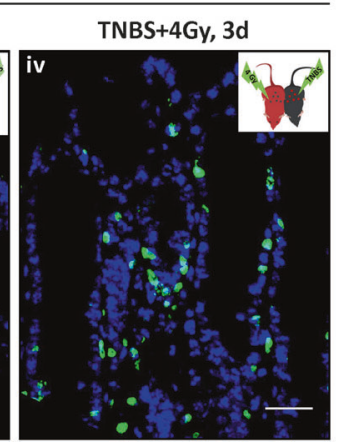

Fig. 8 Depletion of the bone marrow of the donor parabiotic partner reverses its protective effects on mucosal healing in TNBS-induced colitis. a Body weight change in $2.5 \mathrm{mg}$ TNBS-received parabiont mice without radiation $(n=14$ pairs) and with prior $4 \mathrm{~Gy}$ radiation ( $n=5$ pairs) to the donor mouse. Data are presented as means \pm SEM. b (i) Macroscopic images of colons from 2.5 mg TNBS-received parabiont mice without radiation and with prior $4 \mathrm{~Gy}$ radiation. (ii) Colon length of $2.5 \mathrm{mg}$ TNBS-received parabiont mice without radiation $(n=6-8$ pairs as indicated) and with prior 4 Gy radiation ( $n=7$ pairs). Data are presented as means \pm SEM. c Representative H\&E-stained sections from TNBSreceived parabiont mice without radiation (i and ii) and with prior $4 \mathrm{~Gy}$ radiation (iii and iv); Representative Alcian blue-stained sections from TNBS-received parabiont mice without radiation ( $\mathrm{v}$ and vi) and with prior $4 \mathrm{~Gy}$ radiation (vii and viii). Original magnification $\times 20$; scale bars $100 \mu \mathrm{m}$. (ix) Histological damage level of $2.5 \mathrm{mg}$ TNBS-treated parabionts with or without $4 \mathrm{~Gy}$ radiation was scored in a blind manner. Parabiont mouse without radiation ( $n=6$ pairs) and parabiont mouse with 4 Gy radiation ( $n=7$ pairs) from TNBS injury model. Data are presented as means \pm SEM. d RT-qPCR of cytokines in $2.5 \mathrm{mg}$ TNBS-received parabiont mice without radiation $(n=5-6$ pairs as indicated) and with prior $4 \mathrm{~Gy}$ radiation ( $n=7$ pairs) (i-iii). Data are shown as means \pm SEM. e Representative immunohistochemistry images of TUNEL from $2.5 \mathrm{mg}$ TNBS-received parabiont mice with or without radiation (i and ii); representative immunohistochemistry images of BrdU from 2.5 mg TNBS-received parabiont mice with or without radiation (iii-iv). Original magnification $\times 40$; scale bars $50 \mu \mathrm{m}\left({ }^{*} p<0.05\right.$; ${ }^{* *} p<0.01$; ${ }^{* * *} p<0.001$; $* * * * 0<0.0001)$ 
Enteric irradiation and TNBS-induced colitis

One month after the development of a shared circulation in parabiotic pairs, mice were subjected to the following models of intestinal injury:

1. Irradiation injury to the intestine: Mice were exposed to wholebody gamma irradiation after mice were anesthetized by intramuscular injection with a solution consisting of $100 \mathrm{mg} / \mathrm{kg}$ ketamine and $15 \mathrm{mg} / \mathrm{kg}$ xylazine. A Gammacell 40 Exactor (a dual Caesium137, MDS Nordion) was used to expose mice to 8 or $12 \mathrm{~Gy}$ whole body irradiation, with lead shielding applied to protect the non-irradiated parabiont pair as a control. Intestinal samples were harvested 3 days later. Histologic evaluation of the intestine by a pathologist (L.V.) blinded to the groups revealed the presence of intestinal mucosal sloughing, leukocyte infiltration, and crypt apoptosis.

2. Induction of TNBS colitis: In parallel studies, chemical colitis was induced according to the protocol of Wirtz, S. et al. ${ }^{23}$ in which mice were pre-sensitized by applying $1.523 \mathrm{mg}$ of 2,4,6-Trinitrobenzenesulfonic acid solution (TNBS, Sigma-Aldrich, St. Louis, MO) to the skin. On day 8, mice that had been fasted for $12 \mathrm{~h}$ were anesthetized by isoflurane inhalation and then $2.5 \mathrm{mg}$ of TNBS/ $100 \mu \mathrm{l}$ of $50 \%(\mathrm{v} / \mathrm{v})$ ethanol solution was instilled rectally by using a 3.5 French catheter. Mice were kept in a vertical position with the head down for 3 min to allow for efficient delivery of TNBS to the distal colon. Control mice were administered with $100 \mu \mathrm{l}$ of PBS in a similar manner. Mice were then checked daily with respect to their general condition, body weight, consistency of stools, and occult blood in stools.

To evaluate the role of bone marrow-derived cells in the healing response from injury, ablation of the bone marrow was achieved by subjecting the control pair in the TNBS colitis injury model to 4 Gy gamma radiation 4 days prior to instillation of the TNBS.

Bone marrow cell transplant and flow cytometry

In order to evaluate the effects of circulating bone marrowderived cells in the healing response to intestinal mucosal injury, we first harvested the bone marrow from the long bones of tdTomato-CAG mice, and then injected total bone marrow cells or magnetic-sorted $\mathrm{CD}_{4} 5^{+}$or $\mathrm{CD}_{4} 5^{-}$bone marrow cells (as described below) into an unpaired wild-type recipient which had been exposed to 8 or $12 \mathrm{~Gy}$ gamma irradiation or $2.5 \mathrm{mg}$ of TNBS $24 \mathrm{~h}$ earlier. To select CD45 ${ }^{+}$bone marrow cells from the bone marrow, harvested bone marrow cells were magnetically labeled with CD45 MicroBeads (Miltenyi Biotec), and enriched by using LS MACS columns (Miltenyi Biotec). The unlabeled cell fraction was collected as $\mathrm{CD}_{4} 5^{-}$bone marrow cells. The presence of transplanted tdTomato $^{+}$in the intestine was then assessed by fluorescent microscopy. To assess the depletion of bone marrow cells in 4Gy-radiated mice, the bone marrow cavity in the femurs and tibias of wild type mice were flushed with PBS and collected. After removing red blood cells by incubation in lysis buffer, cells were passed through a $40-\mu \mathrm{m}$ cell strainer to obtain a single cell suspension. After centrifugation at $400 \times g$ at $4{ }^{\circ} \mathrm{C}$ for $5 \mathrm{~min}$, the pellet was re-suspended in FACS buffer and centrifuged at $400 \times g$ at $4{ }^{\circ} \mathrm{C}$ for $5 \mathrm{~min}$ and the supernatant was then discarded. For cell surface marker staining, single-cell suspensions were then incubated with anti-CD16/CD32 (BD Biosciences) to block Fc receptor binding $\left(20 \mathrm{~min}, 4^{\circ} \mathrm{C}\right)$. Cells were then pelleted by centrifugation and resuspended in primary fluorochrome-conjugated antibody: CD45, Sca-1, c-kit, and CXCR4 (Biolegend) in FACS buffer and assessed on a BD Accuri C6 or BD LSRFortessa flow cytometer and analyzed using FlowJo software.

Characterization of cellular identity in small intestine by flow cytometry

To evaluate the identity of the migrated cells of donor origin in the injured parabiont lamina propria, the entire small intestine was harvested and cut into $0.5 \mathrm{~cm}$ pieces, followed by incubation in Hank's salt solution without $\mathrm{Mg}^{2+}$ and $\mathrm{Ca}^{2+}$ containing $5 \mathrm{mM}$ EDTA, $1 \mathrm{mM}$ DTT, and $5 \%$ FBS in a $37^{\circ} \mathrm{C}$ shaker at $200 \mathrm{rpm}$. Following stirring, the tissue was strained from the supernatant through a metal sieve, then incubated in enzymatic solution containing $5 \%$ FBS, $0.5 \mathrm{mg} / \mathrm{ml}$ Collagenase D, $0.5 \mathrm{mg} / \mathrm{ml}$ DNase I, and $3 \mathrm{mg} / \mathrm{ml}$ Dispase II in a $37^{\circ} \mathrm{C}$ shaker at $200 \mathrm{rpm}$ and mechanically dissociated via the gentleMACS Dissociator (Miltenyi Biotec) to obtain single cell suspension. Cells were then stained using primary fluorochrome-conjugated antibody: CD45, Sca-1, CXCR4, and c-kit (Biolegend) and assessed on a BD LSRFortessa flow cytometer and analyzed using FlowJo software.

Histology and histopathological scoring

Evidence of intestinal mucosal injury was quantified $(0=$ none, $1=$ mild, $2=$ moderate, $3=$ high) in H\&E-stained sections of small intestine and colon by a pathologist (L.V.) blinded to the treatment group as described Supplementary Tables 2 and 3. The severity of radiation enteritis was assessed by the degree of preservation of the epithelial architecture, glandular dropout, prominent Paneth cells, and infiltration of inflammatory cells in the lamina propria. In TBNS-induced colitis, colonic injury was assessed based on the degree of epithelial injury, goblet cell phenotype, glandular dropout, inflammatory lamina propria expansion, ulceration, pseudomembrane, ischemic changes, and mucosal erosion. For goblet cell staining, paraffin sections were stained with Alcian blue solution and counterstained with nuclear fast red solution (Sigma-Aldrich, St. Louis, MO). For "Swiss roll" sections, the intestine was cut longitudinally and rolled around a toothpick. Tissues were fixed overnight in $4 \%$ paraformaldehyde at $4{ }^{\circ} \mathrm{C}$ prior to staining.

Immunohistochemical staining

Immunohistochemical staining was performed on 5-14 $\mu$ m-thick paraffin embedded or cryosections as follows. For cryosections, slides were thawed at room temperature and rehydrated with PBS. Paraffin sections were first warmed to $63^{\circ} \mathrm{C}$ in a vacuum incubator (Isotemp Vacuum Oven, Fisher Scientific) then washed immediately twice in xylene, gradually re-dehydrated in ethanol $100 \%$, $95 \%, 70 \%$, water), and then processed for antigen retrieval in citrate buffer $(10 \mathrm{mM} \mathrm{pH} \mathrm{6.0)/microwave} \mathrm{(1000} \mathrm{W,} 6 \mathrm{~min})$. For bromdeoxyuridine (BrdU) staining, samples from mice injected with BrdU labeling reagent (Invitrogen) were incubated in $2 \mathrm{M}$ hydrochloric acid $(\mathrm{HCl})$ solution (40 min, room temperature) before the blocking step. Samples were then washed with PBS, blocked with $1 \% \mathrm{BSA} / 5 \%$ donkey-serum ( $1 \mathrm{~h}$, room temperature), then incubated overnight at $4{ }^{\circ} \mathrm{C}$ with primary antibodies $(1: 200$ dilutions in $0.5 \%$ BSA), washed three times with PBS, incubated with appropriate fluorescent-labeled secondary antibodies (1:1000 dilution in $0.5 \%$ BSA, Life Technologies Inc.). To assess apoptosis, samples were incubated with terminal deoxynucleotidyl transferase dUTP nick end labeling (TUNEL) detection solution (In Situ Cell Death Detection Kit, TMR red, Roche) for $1 \mathrm{~h}$ at $37^{\circ} \mathrm{C}$ after the addition of secondary antibody. Slides were incubated with the nuclear marker DAPI (Biolegend), followed by multiple washes, and slides were then mounted using Gelvatol (Sigma-Aldrich) solution prior to imaging using a Nikon Eclipse Ti Confocal microscope under appropriate filter sets.

\section{Quantification of fluorescence intensity}

Fluorescence images were acquired with a Nikon Eclipse Ti Confocal microscope. Randomly selected sections were immunostained following strictly identical immunohistochemistry protocols. Measurements of fluorescence staining intensity was carried out with NIH ImageJ by converting color images to binary scale (fluorescence intensity from 0 to 255), and mean intensity in a region of interest was calculated. Intensity was analyzed in 3-9 regions of the tissue in a single section per animal. 
Quantitative real-time PCR

Total RNA was extracted from the small intestine or colon using RNeasy Mini Kit (Qiagen). $0.5 \mu \mathrm{g}$ of each RNA sample was reverse transcribed into cDNA using the QuantiTect Reverse Transcription Kit (Qiagen) and quantitative real-time PCR was performed using iTaq UniverSYBR Green SMX 5000 (Biodrad) in the Bio-Rad CFX96 Real-Time System (Bio-Rad) with the primers listed in Supplementary Table 4 relative to the housekeeping gene ribosomal protein large, PO (RPLO).

Effect of anti-MAdCam-1 antibody and CCR2 antagonist on donor cell migration in parabiotic pairs

To assess the potential effects of neutralizing anti-MAdCam-1 antibody and CCR2 antagonist on donor cell migration in parabiotic pairs in the setting of TNBS-induced colitis, parabiotic pairs were exposed to TNBS injury as described above. Anti-mouse MAdCam-1 antibody (Biolegend) or CCR2 antagonist (RS 504393, $R \& D$ system) were administered i.p. at dose of 10 and $2 \mathrm{mg} / \mathrm{kg} /$ day, respectively, on day 1 and day 2 post-TNBS instillation. For control group, species-matched lg isotype (Rat lgG) or DMSO was administered to TNBS-colitis parabiosis mice.

Statistical analysis

Data are shown as means \pm SEM and were analyzed using onetailed Student's $t$ tests for comparisons between two groups, oneway analysis of variance (ANOVA) with Sidak's test for multiple comparisons using PRISM version 7.0 (GraphPad). $p<0.05$ was considered to be significant for all experiments.

\section{ACKNOWLEDGEMENTS}

D.J.H. is supported by R01GM078238 and R01DK083752 from the National Institutes of Health and the Robert James Garrett Fund for the Surgical Treatment of Children from the Johns Hopkins University.

\section{AUTHOR CONTRIBUTIONS}

Conceptualization, JS, CPS, and DJH; Methodology, JS, CPS, and DJH; Investigation, JS, $\mathrm{CPS}, \mathrm{XH}, \mathrm{LV}, \mathrm{DC}, \mathrm{HJ}$, and QA; Writing-Original Draft, JS; Writing-Review and Editing, JS, CPS and DJH; Funding acquisition, DJH; Resources, DJH; Supervision, DJH.

\section{ADDITIONAL INFORMATION}

The online version of this article (https://doi.org/10.1038/s41385-018-0123-3) contains supplementary material, which is available to authorized users.

Competing interests: The authors declare no competing interests.

Publisher's note: Springer Nature remains neutral with regard to jurisdictional claims in published maps and institutional affiliations.

\section{REFERENCES}

1. Maloy, K. J. \& Powrie, F. Intestinal homeostasis and its breakdown in inflammatory bowel disease. Nature 474, 298-306 (2011).

2. lizuka, M. \& Konno, S. Wound healing of intestinal epithelial cells. World J. Gastroenterol. 17, 2161-2171 (2011).

3. Umar, S. Intestinal tem cells. Curr. Gastroenterol. Rep. 12, 340-348 (2011).

4. Tian, $H$. et al. A reserve stem cell population in small intestine renders Lgr5positive cells dispensable. Nature v, 265-275 (2012).

5. Van Es, J. H. et al. Dll1+secretory progenitor cells revert to stem cells upon crypt damage. Nat. Cell Biol. 14, 1099-1104 (2012).

6. Tetteh, P. W. et al. Replacement of lost Lgr5-positive stem cells through plasticity of their enterocyte-lineage daughters. Cell Stem Cell 18, 203-213 (2016).

7. Brittan, M. et al. A regenerative role for bone marrow following experimental colitis: contribution to neovasculogenesis and myofibroblasts. Gastroenterology 128, 1984-1995 (2005).

8. Chang, P.-Y., Qu, Y.-Q., Wang, J. \& Dong, L.-H. The potential of mesenchymal stem cells in the management of radiation enteropathy. Cell Death Dis. 6, e1840 (2015).

9. Mortazavi, S. M. J. et al. The healing effect of bone marrow-derived stem cells in acute radiation syndrome. Pak. J. Med. Sci. 32, 646-651 (2016).
10. Lane, S. W., Williams, Da \& Watt, F. M. Modulating the stem cell niche for tissue regeneration. Nat. Biotechnol. 32, 795-803 (2014).

11. Stzepourginski, l. et al. CD34 ${ }^{+}$mesenchymal cells are a major component of the intestinal stem cells niche at homeostasis and after injury. Proc. Natl Acad. Sci. USA 114, E506-E513 (2017).

12. $\mathrm{Xu}, \mathrm{L}$. \& Li, G. Circulating mesenchymal stem cells and their clinical implications. J. Orthop. Transl. 2, 1-7 (2014).

13. Piscaglia, A. C. et al. Circulating hematopoietic stem cells and putative intestinal stem cells in coeliac disease. J. Transl. Med. 13, 220 (2015).

14. Rizvi, A. Z. et al. Bone marrow-derived cells fuse with normal and transformed intestinal stem cells. Proc. Natl Acad. Sci. USA 103, 6321-6325 (2006).

15. Hoogduijn, M. J. et al. No evidence for circulating mesenchymal stem cells in patients with organ injury. Stem Cells Dev. 23, 2328-2335 (2014).

16. Jong, J. Hde et al. Fusion of intestinal epithelial cells with bone marrow derived cells is dispensable for tissue homeostasis. Sci. Rep. 2, 1-7 (2012).

17. Kudo, K. et al. Transplantation of mesenchymal stem cells to prevent radiationinduced intestinal injury in mice. J. Radiat. Res. 51, 73-9 (2010).

18. Chalovich, J. M. \& Eisenberg, E. Use of the parabiotic model in studies of cutaneous wound healing to define participation of circulating cells. Biophys. Chem. 257, 2432-2437 (2005).

19. Herington, J. L. et al. Cross-circulation and cell distribution kinetics in parabiotic. Mice 95, 1295-1301 (2012)

20. Castellano, J. M. et al. In vivo assessment of behavioral recovery and circulatory exchange in the peritoneal parabiosis model. Sci. Rep. 6, 29015 (2016).

21. $\mathrm{Yu}, \mathrm{J}$. Intestinal stem cell injury and protection during cancer therapy. Transl. Cancer Res. 2, 384-396 (2013).

22. Antoniou, E. et al. The TNBS-induced colitis animal model: an overview. Ann. Med. Surg. 11, 9-15 (2016).

23. Wirtz, S., Neufert, C., Weigmann, B. \& Neurath, M. F. Chemically induced mouse models of intestinal inflammation. Nat. Protoc. 2, 541-546 (2007).

24. Ong, Z. Y. et al. Pro-inflammatory cytokines play a key role in the development of radiotherapy-induced gastrointestinal mucositis. Radiat. Oncol. 5, 22 (online journal open access) (2010).

25. Atreya, R. \& Neurath, M. F. Chemokines in inflammatory bowel diseases. Dig. Dis. 28, 386-394 (2010).

26. Scheiffele, F. \& Fuss, I. J. Induction of TNBS colitis in mice. Curr. Protoc. Immunol. 1-14 (2002). https://doi.org/10.1002/0471142735.im1519s49

27. Becciolini, A., Fabbrica, D., Cremonini, D. \& Balzi, M. Quantitative changes in the goblet cells of the rat small intestine after irradiation. Acta Radiol. Oncol. 24, 291-299 (1985)

28. Erben, U. et al. A guide to histomorphological evaluation of intestinal inflammation in mouse models. Int. J. Clin. Exp. Pathol. 7, 4557-4576 (2014).

29. Schnoor, M. E-cadherin is important for the maintenance of intestinal epithelial homeostasis under basal and inflammatory conditions. Dig. Dis. Sci. 60, 816-818 (2015).

30. Andreyev, H. J. N., Wotherspoon, A., Denham, J. W. \& Hauer-Jensen, M. Defining pelvic-radiation disease for the survivorship era. Lancet Oncol. 11, 310-312 (2010).

31. Hauer-Jensen M, Denham JW, Andreyev HJ. Radiation enteropathy-pathogenesis, treatment and prevention. Nat. Rev. Gastroenterol. Hepatol. 11, 470-479 (2014).

32. Bentzen, S. M. Preventing or reducing late side effects of radiation therapy: radiobiology meets molecular pathology. Nat. Rev. Cancer 6, 702-713 (2006).

33. Stacey, R. \& Green, J. T. Radiation-induced small bowel disease: Latest developments and clinical guidance. Ther. Adv. Chronic Dis. 5, 15-29 (2014).

34. Sandborn, W. J. The present and future of inflammatory bowel disease treatment. Gastroenterol. Hepatol. 12, 438-441 (2016).

35. Irvine, E. J. Review article: patients' fears and unmet needs in inflammatory bowel disease. Aliment. Pharmacol. Ther. 20, 54-9 (2004).

36. Craig, R. M., Traynor, A., Oyama, Y. \& Burt, R. K. Hematopoietic stem cell transplantation for severe Crohn's disease. Bone Marrow Transplant. 32, S57-S59 (2003).

37. Wu, J. M. F. et al. Circulating cells contribute to cardiomyocyte regeneration after injury. Circ. Res. 116, 633-641 (2015).

38. Yamasaki, S. et al. Circulating nucleated peripheral blood cells contribute to earlyphase meniscal healing. J. Tissue Eng. Regen. Med. 11, 609-617 (2017).

39. $\mathrm{Li}, \mathrm{C}$. et al. RhoA determines lineage fate of mesenchymal stem cells by modulating CTGF-VEGF complex in extracellular matrix. Nat. Commun. 7, 11455 (2016).

40. Chang, P. et al. Multi-therapeutic effects of human adipose-derived mesenchymal stem cells on radiation-induced intestinal injury. Cell Death Dis. 4, e685 (2013).

41. Xiong, J. et al. Effects of interleukin-4 or interleukin-10 gene therapy on trinitrobenzenesulfonic acid-induced murine colitis. BMC 13, 1-7 (2013).

42. Sémont, a et al. Mesenchymal stem cells improve small intestinal integrity through regulation of endogenous epithelial cell homeostasis. Cell Death Differ. 17, 952-961 (2010). 
The recruitment of extra-intestinal cells to the injured mucosa promotes... $J$ Sung et al.

43. Ando, Y. et al. Subcutaneous adipose tissue-derived stem cells facilitate colonic mucosal recovery from 2,4,6-trinitrobenzene sulfonic acid (TNBS)-induced colitis in rats. Inflamm. Bowel Dis. 14, 826-838 (2008).

44. Procaccino, F. et al. Protective effect of epidermal growth factor in an experimental model of colitis in rats. Gastroenterology 107, 12-7 (1994).

45. Boerma, M., Wang, J., Sridharan, V., Herbert, J. M. \& Hauer-Jensen, M. Pharmacological induction of transforming growth factor-beta1 in rat models enhances radiation injury in the intestine and the heart. PLOS ONE 8, e70479 (2013).
46. Tomita, T. et al. Systemic, but not intestinal, IL-7 is essential for the persistence of chronic colitis. J. Immunol. 180, 383-390 (2008).

47. Tomita, $T$. et al. Colitogenic CD4+effector-memory $T$ cells actively recirculate in chronic colitic mice. Inflamm. Bowel Dis. 14, 1630-1640 (2008).

48. Ono, Y. et al. T-helper 17 and interleukin-17-producing lymphoid tissue inducerlike cells make different contributions to colitis in mice. Gastroenterology 143 1288-1297 (2012).

49. Mikami, Y. et al. Competition between colitogenic Th1 and Th17 cells contributes to the amelioration of colitis. Eur. J. Immunol. 40, 2409-2422 (2010). 